\title{
Conservation of Forestry Biomass with the Use of Alternative Resource
}

\author{
Manju Agarwal, Rachana Pathak \\ Department of Mathematics \& Astronomy, Lucknow University, Lucknow, India \\ Email: manjuak@yahoo.com, rachanapathak2@gmail.com
}

Received 30 November 2014; accepted 25 March 2015; published 1 April 2015

Copyright (C) 2015 by authors and Scientific Research Publishing Inc.

This work is licensed under the Creative Commons Attribution International License (CC BY). http://creativecommons.org/licenses/by/4.0/

\begin{abstract}
The effect of the alternative resource and time delay on conservation of forestry biomass is studied by considering a nonlinear mathematical model. In this paper, interaction between forestry biomass, industrialization pressure, toxicant pressure and technological effort is proposed and analysed. We find out the critical value of delay and observe that there is Hopf bifurcation. Using the normal form theory and the center manifold theorem, we determine the stability and direction of the bifurcating periodic solutions. Numerical simulations are given to illustrate the analytical results.
\end{abstract}

\section{Keywords}

Forestry Biomass, Industrialization, Alternative Resource, Toxicant, Technological Effort, Local Stability, Hopf Bifurcation

\section{Introduction}

Forest is an integral part of our biosphere. It used for fuel, furniture etc. and thus provides strong foundation for the development of any country. Forest assists in the global cycling of water, oxygen, carbon and nitrogen. In many developing countries, people burn wood to get energy for heating and cooking. Forest also provides food and shelter to many wild life species. Due to overpopulation, industrialization and associated pollution forests are depleted alarmingly. A typical example is the Doon Valley in the northern part of India where the forestry resources are being depleted by limestone quarries, wood and paper based industries, growth of human and livestock populations, expansion of forest land for agriculture and settlement etc., threatening the ecological stability of the entire region [1]. It is therefore required a suitable harvesting plan to keep ecological balance. For controlling depletion of forestry biomass, alternative resources like synthetic, liquid wood, plastic, wood composite lumber etc. can play an important part. The following examples also motivate us to consider biomass- 
industry system with alternative resource.

1) To overcome the worldwide problem of conservation of forestry resources, synthetic is a good alternative of wood based product as it is cheap, and needs not much maintenance, and the one most important thing is that it looks fresher than wood based products.

2) Plastic and wood composite lumber are quickly becoming a common replacement for redwood, cedar, and treated lumber in such applications as decking, door and window frames, and exterior moldings. Redwood and cedar decking use virgin trees, maintaining our dependence on scarce wood resources. Plastic and wood composite lumber are worked similarly to real wood and do not require treatment, yet they hold up well to water, sun, insects, and salt air, typical enemies of wood [2].

[3] proposed and analyzed a mathematical model for the survival of a resource-dependent biological population (such as human beings) where both the population and its resource were affected by a toxicant emitted into the environment from external sources as well as formed by its precursors. [4] investigated a nonlinear mathematical model to study the depletion of forestry resources caused by population and population pressure augmented industrialization. It is shown that the equilibrium density of resource biomass decreases as the equilibrium densities of population and industrialization increase. It is found that even if the growth of population (whether intrinsic or by migration) is only partially dependent on resource, still the resource biomass is doomed to extinction due to large population pressure augmented industrialization. It is noted that for sustained industrialization, control measures on its growth are required to maintain the ecological stability. In [5], they proposed a nonlinear mathematical model and analyzed to study the survival of resource-dependent competing species. It is assumed that competing species and its resource are affected simultaneously by a toxicant emitted into the environment from external sources as well as formed by precursors of competing species. It is concluded from the analysis that as the cumulative rates of emission and formation of toxicants into the environment increase, the densities of both competing species and its resource decrease. [6] studied the effect of alternative resource (synthetic) on the conservation of forestry biomass which grew logistically decays due to presence of wood based industries.

In same year, [7] studied the effect of time delay on conservation of forestry biomass by proposing a nonlinear mathematical model. They assumed that the density of forestry biomass depleted due to the presence of human population and it was being conserved by applying some technological efforts. Further, [8] and [9] investigated and concluded a nonlinear mathematical model to study the depletion of forest resources caused by population and the corresponding population pressure.

As a consequence, we propose a model for the interaction of forestry biomass with industrialization pressure, toxicant pressure and applied technological effort. Further, the effect of alternative resource on the growth of forestry biomass is seen. The time delay is the inherent property of the dynamical systems and plays an important role in almost all branches of science and particularly in the biological sciences. In the further study of the model, we see the effect of time delay on the growth rate of forestry biomass. The rest of this paper is organized as follows: In Section 2, we analyze our model with regard to equilibria and their positive conditions. In Section 3, we investigate the stability of positive equilibrium and stability and direction of Hopf bifurcation. In Section 4, some numerical supports are carried out to justify the analytic results obtained in the manuscript. Section 5 deals with the conclusions of the paper.

\section{Mathematical Model}

We consider the following system of differential equations:

$$
\begin{aligned}
& \frac{\mathrm{d} B}{\mathrm{~d} t}=r B\left(1-\frac{B}{K}\right)-\frac{\alpha_{1} B I}{1+B}, \\
& \frac{\mathrm{d} I}{\mathrm{~d} t}=r_{1} I\left(1-\frac{I}{L}\right)+\frac{\alpha_{2} B I}{1+B},
\end{aligned}
$$

where $B(0)=B_{0} \geq 0, \quad I(0)=I_{0} \geq 0$.

In model system (1), $B(t)$ and $I(t)$ are the concentration of forestry biomass and industries, respectively. $r$ and $K$ are intrinsic growth rate and carrying capacity of biomass and $r_{1}, L$ are intrinsic growth and carrying capacity of industries, respectively. $\alpha_{1}$ and $\alpha_{2}$ represents the depletion rate of forest biomass and growth rate of industries in presence of forestry biomass. In the above system (1), growths of industries are 
based on forestry biomass. For controlling depletion of forestry biomass, alternative resources can play an important role. Using alternative resource $\left(A_{r}\right)$, the model (1) can be formulated as

$$
\begin{aligned}
& \frac{\mathrm{d} B}{\mathrm{~d} t}=r B\left(1-\frac{B}{K}\right)-\frac{\alpha_{1} B I A_{r}}{1+B}, \\
& \frac{\mathrm{d} I}{\mathrm{~d} t}=r_{1} I\left(1-\frac{I}{L}\right)+\frac{\alpha_{2} B I A_{r}}{1+B}+\left(1-A_{r}\right) I,
\end{aligned}
$$

where $B(0)=B_{0} \geq 0, I(0)=I_{0} \geq 0$.

Here $A_{r}$ is a time independent constant and its origin is the alternative resource. If $A_{r}=1$, the industries depend only on the forestry biomass and thus it is clear that the system (1) is special case of system (2). If $A_{r}=0$ then both the forestry biomass and industries grow without any interaction. In such case, the industries pressure on forestry biomass is completely removed and industries evolve in presence of alternative food only. But such decoupled system is out of our interest. For neglecting above both cases, $A_{r}$ always lies between 0 and 1 in our system. Due to advancement in technology and industrialization at rapid pace, large amount of toxicants enter into both aquatic ant terrestrial environment and affect biomass. Let us assume that $T(t)$ is the concentration of toxicant in the environment at time $t$. Emission of toxicant into the environment from various external sources and industries is $Q_{0}$ and $Q_{1}$. The constant $\delta_{0}$ is the natural washout rate coefficient of toxicant present in the environment, $\gamma_{1}$ and $\gamma_{2}$ are the depletion rate coefficients of toxicant concentration in the environment due to its uptake by the forestry biomass. After adding this in system (2), our extend model is as follows:

$$
\begin{aligned}
& \frac{\mathrm{d} B}{\mathrm{~d} t}=r B\left(1-\frac{B}{K}\right)-\frac{\alpha_{1} B I A_{r}}{1+B}-\gamma_{1} B T, \\
& \frac{\mathrm{d} I}{\mathrm{~d} t}=r_{1} I\left(1-\frac{I}{L}\right)+\frac{\alpha_{2} B I A_{r}}{1+B}+\left(1-A_{r}\right) I, \\
& \frac{\mathrm{d} T}{\mathrm{~d} t}=Q(I)-\delta_{0} T-\gamma_{2} B T .
\end{aligned}
$$

Here $Q(I)=Q_{0}+Q_{1} I$.

Where $B(0)=B_{0} \geq 0, I(0)=I_{0} \geq 0, T(0)=T_{0} \geq 0$.

The system (3) is further modified when the technological effort $\left(T_{c}\right)$ is applied to conserve the biomass. Thus system (3) become as:

$$
\begin{aligned}
\frac{\mathrm{d} B}{\mathrm{~d} t} & =r B\left(1-\frac{B}{K}\right)-\frac{\alpha_{1} B I A_{r}}{1+B}-\gamma_{1} B T+\phi_{1} B T_{c}, \\
\frac{\mathrm{d} I}{\mathrm{~d} t} & =r_{1} I\left(1-\frac{I}{L}\right)+\frac{\alpha_{2} B I A_{r}}{1+B}+\left(1-A_{r}\right) I, \\
\frac{\mathrm{d} T}{\mathrm{~d} t} & =Q(I)-\delta_{0} T-\gamma_{2} B T, \\
\frac{\mathrm{d} T_{c}}{\mathrm{~d} t} & =\phi(K-B(t-\tau))-\phi_{0} T_{c}
\end{aligned}
$$

where $B(\gamma)=B_{0} \geq 0$ for $\gamma \in[-\tau, 0], I(0)=I_{0} \geq 0, T(0)=T_{0} \geq 0, T_{c}(0)=T_{c 0} \geq 0$.

Here $T_{c}$ is the measure of effort due to technology applied for conservation of forestry and $\phi_{1}$ is the growth rate coefficient of forestry biomass due to technological effort. The constant $\phi$ is the growth-rate coefficient of technological efforts and $\phi_{0}$ is the natural depletion-rate coefficient of technological effort.

Lemma: The region of attraction for the model system (4) is given by the set:

$$
\Omega=\left\{\left(B, I, T, T_{c}\right): 0 \leq B \leq B_{m}, 0 \leq I \leq I_{m}, 0 \leq T \leq T_{m}, 0 \leq T_{c} \leq T_{c_{m}}\right\}
$$

where $B_{m}=\frac{K\left(r \phi_{0}+\phi_{1} \phi K\right)}{\phi_{0} r}, I_{m}=\frac{L\left(r_{1}+1-A_{r}+\alpha_{2} B_{m} A_{r}\right)}{r_{1}}, T_{m}=\frac{Q_{0}+Q_{1} I_{m}}{\delta_{0}}$ and $T_{c_{m}}=\frac{\phi K}{\phi_{0}}$ and it attracts all solutions initiating in the interior of the positive octant. 
Equilibrium analysis: It can be checked that system (4) has four nonnegative equilibria namely, $E_{0}\left(0,0, \frac{Q_{0}}{\delta_{0}}, \frac{\phi K}{\phi_{0}}\right), E_{1}\left(\bar{B}, 0, \bar{T}, \bar{T}_{c}\right), E_{2}\left(0, \tilde{I}, \tilde{T}, \tilde{T}_{c}\right)$ and $E_{3}\left(B^{*}, I^{*}, T^{*}, T_{c}^{*}\right)$. The existence of the equilibrium point $E_{0}$ is obvious hence omitted. We show the existence of the other equilibria as follows:

Existence of $E_{1}\left(\bar{B}, 0, \bar{T}, \bar{T}_{c}\right)$.

Here $\bar{B}, \bar{T}$ and $\bar{T}_{c}$ are the positive solutions of the following algebraic equations:

$$
\begin{gathered}
r\left(1-\frac{\bar{B}}{K}\right)-\gamma_{1} \bar{T}+\phi_{1} \bar{T}_{c}=0, \\
Q_{0}-\delta_{0} \bar{T}-\gamma_{2} \bar{B} \bar{T}=0, \\
\phi(K-\bar{B})-\phi_{0} \bar{T}_{c}=0 .
\end{gathered}
$$

From Equation (7), we get

$$
\bar{T}_{c}=\frac{\phi(K-\bar{B})}{\phi_{0}} .
$$

Thus, $E_{1}$ exists if: $\bar{B}<K$ which is obvious.

From Equation (6), we get

$$
\bar{T}=\frac{Q_{0}}{\delta_{0}+\gamma_{2} \bar{B}} .
$$

Putting the value of $\bar{T}$ and $\bar{T}_{c}$ in Equation (5), we get

$$
\begin{aligned}
\bar{B} & =\frac{\left(-r \phi_{0} \delta_{0}-\phi_{1} \phi \delta_{0} K+r \phi_{0} \gamma_{2} K+\phi_{1} \phi K^{2} \gamma_{2}\right)}{2\left(\phi_{1} \phi K \gamma_{2}-r \phi_{0} \gamma_{2}\right)} \\
& \pm \frac{\sqrt{\left(r \phi_{0} \delta_{0}+\phi_{1} \phi \delta_{0} K-r \phi_{0} \gamma_{2} K-\phi_{1} \phi K^{2} \gamma_{2}\right)^{2}-4\left(r \phi_{0} \gamma_{2}-\phi_{1} \phi K \gamma_{2}\right)\left(-r \phi_{0} \delta_{0} K+Q_{0} \gamma_{1} \phi_{0} K-\phi_{1} \phi K^{2} \delta_{9}\right)}}{2\left(\phi_{1} \phi K \gamma_{2}-r \phi_{0} \gamma_{2}\right)} .
\end{aligned}
$$

Thus, $E_{2}$ exists if condition $K \gamma_{2}>\delta_{0}$ holds.

Existence of $E_{2}\left(0, \tilde{I}, \tilde{T}, \tilde{T}_{c}\right)$.

Here $\tilde{I}, \tilde{T}$ and $\tilde{T}_{c}$ are the positive solutions of the following algebraic equations:

$$
\begin{gathered}
r_{1}\left(1-\frac{\tilde{I}}{L}\right)+1-A_{r}=0, \\
Q_{0}+Q_{1} \tilde{I}-\delta_{0} \tilde{T}=0, \\
\phi K-\phi_{0} \tilde{T}_{c}=0 .
\end{gathered}
$$

From Equation (10), we get

$$
\tilde{T}_{c}=\frac{\phi K}{\phi_{0}}
$$

From Equation (8), we get

$$
\tilde{I}=\frac{L\left(r_{1}+1-A_{r}\right)}{r_{1}} .
$$

Putting the value of $\tilde{I}$ in Equation (9), we get 


$$
\tilde{T}=\frac{r_{1} Q_{0}+Q_{1} L\left(r_{1}+1-A_{r}\right)}{\delta_{0}} .
$$

Existence of $E_{3}\left(B^{*}, I^{*}, T^{*}, T_{c}^{*}\right)$.

Here $B^{*}, I^{*}, T^{*}$ and $T_{c}^{*}$ are the positive solutions of the following algebraic equations:

$$
\begin{gathered}
r\left(1-\frac{B^{*}}{K}\right)-\frac{\alpha_{1} I^{*} A_{r}}{1+B^{*}}-\gamma_{1} T^{*}+\phi_{1} T_{c}^{*}=0, \\
r_{1}\left(1-\frac{I^{*}}{L}\right)+\frac{\alpha_{2} B^{*} A_{r}}{1+B^{*}}+\left(1-A_{r}\right)=0, \\
Q_{0}+Q_{1} I^{*}-\delta_{0} T^{*}-\gamma_{2} B^{*} T^{*}=0, \\
\phi\left(K-B^{*}\right)-\phi_{0} T_{c}^{*}=0 .
\end{gathered}
$$

From Equation (14), we get

$$
T_{c}^{*}=\frac{\phi\left(K-B^{*}\right)}{\phi_{0}} .
$$

After simple manipulation, we get from Equation (12) is

$$
I^{*}=\frac{L\left\{r_{1}+1-A_{r}+B^{*}\left(r_{1}+1-A_{r}+\alpha_{2} A_{r}\right)\right\}}{r_{1}\left(1+B^{*}\right)} .
$$

Putting the value of $I^{*}$ in Equation (13), we get

$$
T^{*}=\frac{Q_{0} r_{1}\left(1+B^{*}\right)+Q_{1} L\left\{r_{1}+1-A_{r}+B^{*}\left(r_{1}+1-A_{r}+\alpha_{2} A_{r}\right)\right\}}{r_{1}\left(1+B^{*}\right)\left(\delta_{0}+\gamma_{2} B^{*}\right)} .
$$

Putting the value of $T^{*}, T_{c}^{*}$ and $I^{*}$ in Equation (11), we get

$$
F\left(B^{*}\right)=A_{1}^{*} B^{* 4}+A_{2}^{*} B^{* 3}+A_{3}^{*} B^{* 2}+A_{4}^{*} B^{*}+A_{5}^{*}
$$

where

$$
\begin{aligned}
A_{1}^{*}= & \frac{\gamma_{2} \phi_{0} r r_{1}}{K}+\gamma_{2} \phi_{1} \phi r_{1}, \\
A_{2}^{*}= & \frac{\left(\delta_{0}+\gamma_{2}\right) \phi_{0} r r_{1}}{K}+\frac{r r_{1} \phi_{0} \gamma_{2}}{K}-r r_{1} \phi_{0} \gamma_{2}+\delta_{0} \phi_{1} \phi r_{1}+2 \gamma_{2} \phi_{1} \phi r_{1}-K \gamma_{2} \phi_{1} \phi r_{1}, \\
A_{3}^{*}= & r r_{1} \phi_{0} \delta_{0}\left(\frac{2}{K}+\frac{\gamma_{2}}{\delta_{0} K}-1-\frac{\gamma_{2}}{\delta_{0}}\right)+\gamma_{1} Q_{0} r_{1} \phi_{0}+\gamma_{1} \phi_{0}\left(r_{1}+1-A_{r}+\alpha_{2} A_{r}\right)+\delta_{0} \phi_{1} \phi r_{1}-K \delta_{0} \phi_{1} \phi r_{1}-K \gamma_{2} \phi_{1} \phi r_{1} \\
& -r r_{1} \gamma_{2} \phi_{0}+\phi_{1} \phi r_{1} \delta_{0}+\phi_{1} \phi r_{1} \gamma_{2}-\phi_{1} \phi r_{1} K \gamma_{2}+\alpha_{1} A_{r} L \phi_{0} \gamma_{2}\left(r_{1}+1-A_{r}+\alpha_{2} A_{r}\right), \\
A_{4}^{*}= & r r_{1} \phi_{0} \delta_{0}\left(\frac{1}{K}-2\right)+\gamma_{1}\left(r_{1}+1-A_{r}\right) \phi_{0}-\phi_{1} \phi r_{1}\left(K \delta_{0}+\gamma_{2}\right)+\gamma_{1} Q_{0} r_{1} \phi_{0}+\gamma_{1} \phi_{0}\left(r_{1}+1-A_{r}+\alpha_{2} A_{r}\right) \\
& +\phi_{1} \phi \delta_{0} r_{1}\left(1-K-\frac{K \gamma_{2}}{\delta_{0}}\right)+\alpha_{1} A_{r} L \phi_{0} \gamma_{2}\left(r_{1}+1-A_{r}\right)+\gamma_{1} Q_{0} r_{1} \phi_{0}+\alpha_{1} A_{r} L \phi_{0} \delta_{0}\left(1+r_{1}-A_{r}+\alpha_{2} A_{r}\right), \\
A_{5}^{*}= & \gamma_{2} Q_{0} \phi_{0} r_{1}-r r_{1} \phi_{0} \delta_{0}+\gamma_{1} Q_{0} r_{1} \phi_{0}+\gamma_{1}\left(r_{1}+1-A_{r}\right) \phi_{0}-\phi \phi_{1} r_{1} K \delta_{0}+\alpha_{1} A_{r} L \phi_{0} \delta_{0}\left(r_{1}+1-A_{r}\right) . \\
F(0)= & A_{5}^{*}>0, \text { if } \gamma_{2} Q_{0}>r \delta_{0} ? \quad \gamma_{1} Q_{0} \phi_{0}>\phi \phi_{1} K \delta_{0}, \\
F(K)= & A_{1}^{*} K^{4}+A_{2}^{*} K^{3}+A_{3}^{*} K^{2}+A_{4}^{*} K+A_{5}^{*}<0 .
\end{aligned}
$$

We note that $F(0)>0$ and $F(K)<0$, showing the existence of $B^{*}$ in the interval $0<B^{*}<K$. Now, the 
sufficient condition for $B^{*}$ to be unique positive real is $F^{\prime}\left(B^{*}\right)<0$ at $E_{3}$, where

$$
F^{\prime}\left(B^{*}\right)=4 A_{1}^{*} B^{* 3}+3 A_{2}^{*} B^{* 2}+2 A_{3}^{*} B^{*}+A_{4}^{*}<0 .
$$

Remark 1. From Equation (15), it is easy to note that $\frac{\mathrm{d} B^{*}}{\mathrm{~d} \phi}>0$ and $\frac{\mathrm{d} B^{*}}{\mathrm{~d} A_{r}}>0$, which implies that the equilibrium density of forestry biomass increases as the growth rate coefficient of technological efforts and value of alternative resources increases.

\subsection{Local Stability Analysis without Delay, (i.e. $\tau=0$ )}

To discuss the local stability of system (4), we compute the variational matrix of system (4). The entries of general variational matrix are given by differentiating the right side of system (4) with respect to $B, I, T$ and $T_{c}$ i.e.

$$
V(E)=\left(\begin{array}{cccc}
t_{11} & t_{12} & t_{13} & t_{14} \\
t_{21} & t_{22} & 0 & 0 \\
t_{31} & t_{32} & t_{33} & 0 \\
t_{41} & 0 & 0 & t_{44}
\end{array}\right)
$$

where

$$
\begin{aligned}
& t_{11}=r-\frac{2 r B}{K}-\gamma_{1} T+\phi_{1} T_{c}-\frac{\alpha_{1} I A_{r}}{(1+B)^{2}}, \quad t_{12}=-\frac{\alpha B A_{r}}{1+B}, \quad t_{13}=-\gamma_{1} B, \quad t_{14}=\phi_{1} B, \quad t_{21}=\frac{\alpha_{2} I A_{r}}{(1+B)^{2}}, \\
& t_{22}=r_{1}-\frac{2 r_{1} I}{L}+\frac{\alpha_{2} B A_{r}}{1+B}+1-A_{r}, \quad t_{31}=-\gamma_{2} T, \quad t_{32}=Q_{1}, \quad t_{33}=-\delta_{0}-\gamma_{2} B, \quad t_{41}=-\phi, \quad t_{44}=-\phi_{0} .
\end{aligned}
$$

The variational matrix $V\left(E_{0}\right)$ at equilibrium point $E_{0}$ is given by

$$
V\left(E_{0}\right)=\left(\begin{array}{cccc}
r-\frac{\gamma_{1} Q_{0}}{\delta_{0}}+\frac{\phi_{1} \phi K}{\phi_{0}} & 0 & 0 & 0 \\
0 & r_{1}+1-A_{r} & 0 & 0 \\
-\frac{\gamma_{2} Q_{0}}{\delta_{0}} & Q_{1} & -\delta_{0} & 0 \\
-\phi & 0 & 0 & -\phi_{0}
\end{array}\right) .
$$

The eigenvalues of matrix $V\left(E_{0}\right)$ in the direction of $T$ and $T_{c}$ are negative. So $E_{0}$ has stable manifold in $T-T_{c}$ plane and unstable manifold in $I$-direction. The equilibrium point $E_{0}$ is stable manifold in $B-T-T_{c}$ plane if $\delta_{0}\left(\phi_{0} r+\phi_{1} \phi K\right)>\gamma_{1} Q_{0}$ otherwise it becomes unstable in $B-T-T_{c}$ plane.

The variational matrix $V\left(E_{1}\right)$ at equilibrium point $E_{1}$ is given by

$$
V\left(E_{1}\right)=\left(\begin{array}{cccc}
a_{11} & a_{12} & a_{13} & a_{14} \\
0 & a_{22} & 0 & 0 \\
a_{31} & a_{32} & a_{33} & 0 \\
a_{41} & 0 & 0 & a_{44}
\end{array}\right)
$$

where

$$
\begin{aligned}
& a_{11}=r-\frac{2 r \bar{B}}{K}-\gamma_{1} \bar{T}+\phi_{1} \bar{T}_{c}, \quad a_{12}=-\frac{\alpha \bar{B} A_{r}}{1+\bar{B}}, \quad a_{13}=-\gamma_{1} \bar{B}, \quad a_{14}=\phi_{1} \bar{B}, \\
& a_{22}=r_{1}+\frac{\alpha_{2} \bar{B} A_{r}}{1+\bar{B}}+1-A_{r}, \quad a_{31}=-\gamma_{2} \bar{T}, \quad a_{32}=Q_{1}, \quad a_{33}=-\delta_{0}-\gamma_{2} \bar{B}, \quad a_{41}=-\phi, \quad a_{44}=-\phi_{0} .
\end{aligned}
$$

The characteristic equation corresponding to the variational matrix $V\left(E_{1}\right)$ is given by

$$
\lambda^{4}+C_{1} \lambda^{3}+C_{2} \lambda^{2}+C_{3} \lambda+C_{4}=0,
$$


where

$$
\begin{aligned}
& C_{1}=-a_{11}-a_{22}-a_{33}-a_{44}, \\
& C_{2}=a_{33} a_{44}+a_{22} a_{44}+a_{11} a_{44}+a_{22} a_{33}+a_{11} a_{33}+a_{11} a_{22}-a_{13} a_{31}-a_{14} a_{41}, \\
& C_{3}=-a_{22} a_{33} a_{44}-a_{11} a_{33} a_{44}-a_{11} a_{22} a_{44}-a_{11} a_{22} a_{33}+a_{13} a_{31} a_{44}+a_{22} a_{13} a_{31}+a_{14} a_{41} a_{33}+a_{14} a_{41} a_{22}, \\
& C_{4}=a_{11} a_{22} a_{33} a_{44}-a_{13} a_{31} a_{22} a_{44}-a_{22} a_{33} a_{14} a_{41} .
\end{aligned}
$$

According to Routh-Hurwitz criterion, equilibrium point $E_{1}$ is locally asymptotically stable provided the following conditions are satisfied

$$
C_{1}>0, \quad C_{2}>0, \quad C_{3}>0, \quad C_{4}>0, \quad C_{1} C_{2}-C_{3}>0, \quad C_{3}\left(C_{1} C_{2}-C_{3}\right)-\left(C_{1}\right)^{2} C_{4}>0 .
$$

The variational matrix $V\left(E_{2}\right)$ at equilibrium point $E_{2}$ is given by

where

$$
V\left(E_{2}\right)=\left(\begin{array}{cccc}
b_{11} & 0 & 0 & 0 \\
b_{21} & b_{22} & 0 & 0 \\
b_{31} & b_{32} & b_{33} & 0 \\
b_{41} & 0 & 0 & b_{44}
\end{array}\right)
$$

$$
\begin{aligned}
& b_{11}=r-\gamma_{1}\left[\frac{Q_{0}}{\delta_{0}}+\frac{Q_{1} L\left(r_{1}+1-A_{r}\right)}{r_{1} \delta_{0}}\right]+\frac{\phi_{1} \phi K}{\phi_{0}}-\frac{\alpha_{1} A_{r} L\left(r_{1}+1-A_{r}\right)}{r_{1}}, \\
& b_{21}=\alpha_{2} \tilde{I} A_{r}, \quad b_{22}=-\left(r_{1}+1-A_{r}\right), \\
& b_{31}=-\gamma_{2} \tilde{T}, \quad b_{32}=Q_{1}, \quad b_{33}=-\delta_{0}, \quad b_{41}=-\phi, \quad b_{44}=-\phi_{0} .
\end{aligned}
$$

The variational matrix $V\left(E_{2}\right)$ has four eigenvalues. The sign of three eigenvalues $b_{22}, b_{23}$ and $b_{44}$ are negative so the stability of equilibrium point $E_{2}$ depends on sign of $b_{11}$. The equilibrium point $E_{2}$ is stable manifold in $B-I-T-T_{c}$ plane if $r r_{1} \delta_{0}>\gamma_{1}\left\{Q_{0} r_{1}+Q_{1} L\left(r_{1}+1-A_{r}\right)\right\}$ otherwise it is unstable in $B-I-T-T_{c}$ plane.

The variational matrix $V\left(E_{3}\right)$ at equilibrium point $E_{3}$ is given by

where

$$
V\left(E_{3}\right)=\left(\begin{array}{cccc}
c_{11} & c_{12} & c_{13} & c_{14} \\
c_{21} & c_{22} & 0 & 0 \\
c_{31} & c_{32} & c_{33} & 0 \\
c_{41} & 0 & 0 & c_{44}
\end{array}\right)
$$

$$
\begin{aligned}
& c_{11}=r-\frac{2 r B^{*}}{K}-\gamma_{1} T^{*}+\phi_{1} T_{c}^{*}-\frac{\alpha_{1} I^{*} A_{r}}{\left(1+B^{*}\right)^{2}}, \quad c_{12}=-\frac{\alpha B^{*} A_{r}}{1+B^{*}}, \quad c_{13}=-\gamma_{1} B^{*}, \quad c_{14}=\phi_{1} B^{*}, \quad c_{21}=\frac{\alpha_{2} I^{*} A_{r}}{\left(1+B^{*}\right)^{2}}, \\
& c_{22}=r_{1}-\frac{2 r_{1} I^{*}}{L}+\frac{\alpha_{2} B^{*} A_{r}}{1+B^{*}}+1-A_{r}, \quad c_{31}=-\gamma_{2} T^{*}, \quad c_{32}=Q_{1}, \quad c_{33}=-\delta_{0}-\gamma_{2} B^{*}, \quad c_{41}=-\phi, \quad c_{44}=-\phi_{0} .
\end{aligned}
$$

The characteristic equation corresponding to the variational matrix $V\left(E_{3}\right)$ is given by

$$
\lambda^{4}+G_{1} \lambda^{3}+G_{2} \lambda^{2}+G_{3} \lambda+G_{4}=0
$$

where

$$
\begin{aligned}
G_{1}= & -c_{11}-c_{22}-c_{33}-c_{44}, \\
G_{2}= & c_{33} c_{44}+c_{22} c_{44}+c_{11} c_{44}+c_{22} c_{33}+c_{11} c_{33}+c_{11} c_{22}-c_{13} c_{31}-c_{14} c_{41}-c_{13} c_{31}, \\
G_{3}= & -c_{22} c_{33} c_{44}-c_{11} c_{33} c_{44}-c_{11} c_{22} c_{44}-c_{11} c_{22} c_{33}+c_{12} c_{21} c_{44}+c_{12} c_{21} c_{33}-c_{13} c_{12} c_{32} \\
& +c_{13} c_{31} c_{44}+c_{22} c_{13} c_{31}+c_{14} c_{41} c_{33}+c_{14} c_{41} c_{22}, \\
G_{4}= & c_{11} c_{22} c_{33} c_{44}-c_{12} c_{21} c_{33} c_{44}+c_{13} c_{21} c_{32} c_{44}-c_{13} c_{31} c_{22} c_{44}-c_{22} c_{33} c_{14} c_{41} .
\end{aligned}
$$

According to Routh-Hurwitz criterion, equilibrium point $E_{3}$ is locally asymptotically stable provided the 
following conditions are satisfied

$$
G_{1}>0, \quad G_{2}>0, \quad G_{3}>0, \quad G_{4}>0, \quad G_{1} G_{2}-G_{3}>0, \quad G_{3}\left(G_{1} G_{2}-G_{3}\right)-\left(G_{1}\right)^{2} G_{4}>0 .
$$

\subsection{Local Stability Analysis with Delay, (i.e. $\tau \neq 0$ )}

To discuss the stability behavior of equilibrium $E_{3}$ of system (4) with time delay, (i.e. $\tau \neq 0$ ), we linearize system (4) by using the following transformations:

$$
B=B^{*}+b, \quad I=I^{*}+i, \quad T=T^{*}+a, \quad T_{c}=T_{c}^{*}+c
$$

where $b, i, a$ and $c$ are small perturbations around the equilibrium $E_{3}$.

The linearized system of system (4) about $E_{3}$ is given by:

where $u(t)=[b(t), i(t), a(t), c(t)]$,

$$
\frac{\mathrm{d} u}{\mathrm{~d} t}=M_{1} u(t)+M_{2} u(t-\tau)
$$

$$
M_{1}=\left[\begin{array}{cccc}
-B^{*}\left(\frac{r}{K}-\frac{A_{r} \alpha_{1} I^{*}}{\left(1+B^{*}\right)^{2}}\right) & -\frac{\alpha_{1} B^{*} A_{r}}{1+B^{*}} & -\gamma_{1} B^{*} & \phi_{1} B^{*} \\
\frac{\alpha_{2} A_{r} I^{*}}{1+B^{*}}-\frac{\alpha_{2} A_{r} B^{*} I^{*}}{\left(1+B^{*}\right)^{2}} & -\frac{r_{1} I^{*}}{L} & 0 & 0 \\
-\gamma_{2} T^{*} & Q_{1} & -\left(\delta_{0}+\gamma_{2} B^{*}\right) & 0 \\
0 & 0 & 0 & -\phi_{0}
\end{array}\right]
$$

and

$$
M_{2}=\left[\begin{array}{cccc}
0 & 0 & 0 & 0 \\
0 & 0 & 0 & 0 \\
0 & 0 & 0 & 0 \\
-\phi & 0 & 0 & 0
\end{array}\right] .
$$

The characteristic equation for linearized system (19) is obtained as:

$$
\psi^{4}+p_{1} \psi^{3}+p_{2} \psi^{2}+p_{3} \psi+p\left(q_{1} \psi^{2}+q_{2} \psi+q_{3}\right) \mathrm{e}^{-\psi \tau \tau}=0
$$

where

$$
\begin{gathered}
p_{1}=\phi_{0}+\delta_{0}+\gamma_{2} B^{*}+B^{*}\left(\frac{r}{K}-\frac{A_{r} \alpha_{1} I^{*}}{\left(1+B^{*}\right)^{2}}\right)+\frac{r_{1} I^{*}}{L}, \\
p_{2}=\phi_{0}\left(\delta_{0}+\gamma_{2} B^{*}\right)+\phi_{0}\left[\frac{r_{1} I^{*}}{L}+B^{*}\left(\frac{r}{K}-\frac{A_{r} \alpha_{1} I^{*}}{\left(1+B^{*}\right)^{2}}\right)\right]-\left[\frac{r_{1} I^{*}}{L}+B^{*}\left(\frac{r}{K}-\frac{A_{r} \alpha_{1} I^{*}}{\left(1+B^{*}\right)^{2}}\right)\right]\left(\delta_{0}+\gamma_{2} B^{*}\right) \\
+\frac{r_{1} I^{*}}{L} B^{*}\left(\frac{r}{K}-\frac{A_{r} \alpha_{1} I^{*}}{\left(1+B^{*}\right)^{2}}\right)+\frac{\alpha_{1} \alpha_{2} B^{*} I^{*} A_{r}^{2}}{\left(1+B^{*}\right)^{2}}-\gamma_{2} T^{*} \gamma_{1} B^{*}, \\
p_{3}=\phi_{0}\left[B^{*}\left(\frac{r}{K}-\frac{A_{r} \alpha_{1} I^{*}}{\left(1+B^{*}\right)^{2}}\right)+\frac{r_{1} I^{*}}{L}\right]\left(\delta_{0}+\gamma_{2} B^{*}\right)+\frac{r_{1} I^{*} B^{*}}{L}\left(\frac{r}{K}-\frac{A_{r} \alpha_{1} I^{*}}{\left(1+B^{*}\right)^{2}}\right)\left(\phi_{0}+\delta_{0}+\gamma_{2} B^{*}\right) \\
+\frac{\alpha_{1} \alpha_{2} B^{*} A_{r}^{2} I^{*}}{\left(1+B^{*}\right)^{2}}\left(\phi_{0}+\delta_{0}+\gamma_{2} B^{*}\right)+\frac{\gamma_{1} B^{*} \alpha_{2} A_{r} I^{*} Q_{1}}{1+B^{*}}-\gamma_{1} \gamma_{2} B^{*} T^{*} \phi_{0}-\frac{\gamma_{1} \gamma_{2} r_{1} B^{*} I^{*} T^{*}}{L},
\end{gathered}
$$




$$
\begin{aligned}
p_{4}= & \phi_{0}\left[\left(\delta_{0}+\gamma_{2} B^{*}\right)\left\{\frac{r_{1} I^{*} B^{*}}{L}\left(\frac{r}{K}-\frac{A_{r} \alpha_{1} I^{*}}{\left(1+B^{*}\right)^{2}}\right)+\frac{\alpha_{1} B^{*} A_{r}}{1+B^{*}}\left(\frac{\alpha_{2} A_{r} I^{*}}{1+B^{*}}-\frac{\alpha_{2} A_{r} B^{*} I^{*}}{\left(1+B^{*}\right)^{2}}\right)\right\}\right. \\
& \left.-\gamma_{1} B^{*}\left\{\frac{r_{1} I^{*} \gamma_{2} T^{*}}{L}-Q_{1}\left(\frac{\alpha_{2} A_{r} I^{*}}{1+B^{*}}-\frac{\alpha_{2} A_{r} B^{*} I^{*}}{\left(1+B^{*}\right)^{2}}\right)\right\}\right], \\
q_{1}= & -\phi_{1} \phi B^{*}, \quad q_{2}=-\phi_{1} \phi B^{*}\left(\frac{r_{1} I^{*}}{L}+\delta_{0}+\gamma_{2} B^{*}\right), \quad q_{3}=-\frac{\phi_{1} \phi r_{1} B^{*} I^{*}\left(\delta_{0}+\gamma_{2} B^{*}\right)}{L} .
\end{aligned}
$$

Let $\psi=i \omega$ be one such root. Substituting this in Equation (20) and equating real and imaginary parts, we get

$$
\begin{gathered}
\left(q_{3}+q_{1} \omega^{2}\right) \cos \omega \tau+q_{2} \omega \sin \delta \tau=p_{2} \omega^{2}-\omega^{4}-p_{4}, \\
q_{2} \omega \cos \delta \tau-\left(q_{3}+q_{1} \omega^{2}\right) \sin \delta \tau=p_{1} \omega^{3}+p_{2} \omega .
\end{gathered}
$$

Squaring and adding Equations (21) and (22), we get

$$
\omega^{8}+\bar{D}_{1} \omega^{6}+\bar{D}_{2} \omega^{4}+\bar{D}_{3} \omega^{2}+\bar{D}_{4}=0,
$$

where

$$
\bar{D}_{1}=p_{1}^{2}-2 p_{2}, \quad \bar{D}_{2}=p_{2}^{2}+2 p_{4}+2 p_{1} p_{3}-q_{1}^{2}, \quad \bar{D}_{3}=-2 p_{2} p_{4}+p_{3}^{2}+2 q_{1} q_{3}-q_{2}^{2}, \quad \bar{D}_{4}=p_{4}^{2}-q_{3}^{2} .
$$

Substituting $\omega^{2}=\sigma$ Equation (23) becomes

$$
\begin{gathered}
F(\sigma)=\sigma^{4}+\bar{D}_{1} \sigma^{3}+\bar{D}_{2} \sigma^{2}+\bar{D}_{3} \sigma+\bar{D}_{4}=0, \\
F(0)=\bar{D}_{4}=p_{4}^{2}-q_{3}^{2} .
\end{gathered}
$$

We assume that:

$$
\left(\mathrm{H}_{1}\right): p_{4}<q_{3} .
$$

We notice that $F$ is continuous everywhere with $F(0)<0$ when condition $\left(\mathrm{H}_{1}\right)$ holds and $F(\infty)>0$. Therefore, the Equation (24) always has at least one positive root. Consequently, the stability criteria of the system for $\tau=0$ will not necessarily ensure the stability of the system for $\tau \neq 0$. We assume the Equation (24) has four positive roots denoted by $\sigma_{1}, \sigma_{2}, \sigma_{3}, \sigma_{4}$ denoted as:

$$
\omega_{1}=\sqrt{\sigma_{1}}, \quad \omega_{2}=\sqrt{\sigma_{2}}, \quad \omega_{3}=\sqrt{\sigma_{3}}, \quad \omega_{4}=\sqrt{\sigma_{4}} .
$$

Again solving (21) and (22), we get a critical value of delay given as follows

$$
\tau_{k}=\frac{1}{\omega} \cos ^{-1}\left[\frac{q_{2} \omega\left(p_{1} \omega^{3}+p_{3} \omega\right)+\left(q_{3}-q_{1} \omega^{2}\right)\left(p_{2} \omega^{2}-\omega^{4}-p_{4}\right)}{q_{2}^{2} \omega^{2}+\left(q_{3}-q_{1} \omega^{2}\right)^{2}}\right]+\frac{2 k \pi}{\omega}, \quad k=0,1,2, \cdots .
$$

\subsubsection{Hopf Bifurcation}

To investigate the behavior of the system (4) in the neighborhood of $\tau_{0}$. We represent the following theorem.

Theorem:

We observe that the conditions for Hopf bifurcation are satisfied yielding the required periodic solution, that is,

$$
\left[\frac{\mathrm{d}(\operatorname{Re} \psi)}{\mathrm{d} \tau}\right]_{\tau=\tau_{0}} \neq 0 .
$$

This signifies that there exists at least one eigenvalue with positive real part for $\tau>\tau_{0}$. 
Proof:

Differentiating Equation (20) with respect to $\tau$, we obtain

$$
\left[4 \psi^{3}+3 p_{1} \psi^{2}+2 p_{2} \psi+p_{3}+\mathrm{e}^{-\psi \tau}\left(2 q_{1} \psi+q_{2}\right)-\tau \mathrm{e}^{-\psi \tau}\left(q_{1} \psi^{2}+q_{2} \psi+q_{3}\right)\right] \frac{\mathrm{d} \psi}{\mathrm{d} \tau}=\psi \mathrm{e}^{-\psi \tau}\left(q_{1} \psi^{2}+q_{2} \psi+q_{3}\right) .
$$

Therefore

$$
\left(\frac{\mathrm{d} \psi}{\mathrm{d} \tau}\right)^{-1}=\frac{4 \psi^{3}+3 p_{1} \psi^{2}+2 p_{2} \psi+p_{3}+\mathrm{e}^{-\psi \tau}\left(2 q_{1} \psi+q_{2}\right)}{\psi \mathrm{e}^{-\psi \tau}\left(q_{1} \psi^{2}+q_{2} \psi+q_{3}\right)}-\frac{\tau}{\psi} .
$$

We can obtain here

$$
\left[\frac{\mathrm{d}(\operatorname{Re} \psi)}{\mathrm{d} \tau}\right]_{\tau=\tau_{0}} \neq 0 .
$$

Verifying numerically it has been obtained that the transversality condition holds and hence Hopf bifurcation occurs at $\tau=\tau_{0}$.

\subsubsection{Stability and Direction of Periodic Solutions}

In this section, we will derive explicit formulae for determining the direction, stability and period of the bifurcating periodic solutions arises through Hopf bifurcation. The method we will follow is based on the normal form theory and center manifold theorem as given in [10]. Without loss of generality we denote any of the critical values of $\tau$ by $\tau_{k}$ at which Equation (20) has a pair of purely imaginary roots $\pm i \omega_{0}$ and system undergoes Hopf bifurcation. Hence for any root of Equation (20) of the form $\psi(\tau)=v(\tau)+i \omega(\tau), v\left(\tau_{k}\right)=0$, $\omega\left(\tau_{k}\right)=\omega_{0}$ and $\left|\frac{\mathrm{d} v}{\mathrm{~d} \tau}\right|_{\tau=\tau_{k}} \neq 0$. Let $\tau=\tau_{k}+\mu, \mu \in R$, so that $\mu=0$ is Hopf bifurcation value for the system.

Define the space of continuous real valued functions as $C=C\left([-1,0], R^{4}\right)$. Using the transformation $u_{1}(t)=B(t)-B^{*}, \quad u_{2}(t)=I(t)-I^{*}, \quad u_{3}(t)=T(t)-T^{*}$ and $u_{4}(t)=T_{c}(t)-T_{c}^{*}$ and $\chi_{i}(t)=u_{i}(\tau t)$ for $i=1,2,3,4$; the delay model system (4), then transform to FDE in $C$ as,

$$
\frac{\mathrm{d} \chi}{\mathrm{d} t}=L_{\mu} \chi_{t}+f\left(\mu, \chi_{t}\right)
$$

where $\chi(t)=\left(\chi_{1}(t), \chi_{2}(t), \chi_{3}(t), \chi_{4}(t)\right)^{\mathrm{T}} \in R^{4}, \quad \chi_{t}(\Theta)=\chi(t+\Theta), \quad \Theta \in[-1,0] \quad$ and $\quad L_{\mu}: C \rightarrow R^{4} \quad$ and $f: C \times R \rightarrow R^{4}$ are given by

$$
L_{\mu} \zeta=\left(\tau_{k}+\mu\right)\left[M_{1} \zeta(0)+M_{2} \zeta(-1)\right]
$$

and

$$
f(\mu, \zeta)=\left(\tau_{k}+\mu\right)\left(\begin{array}{c}
Z_{1} \\
Z_{2} \\
Z_{3} \\
0
\end{array}\right)
$$

where

$$
\begin{aligned}
Z_{1}= & \left(\frac{\alpha_{1} A_{r} I^{*}}{\left(1+B^{*}\right)^{2}}-\frac{r}{K}\right) \zeta_{1}^{2}(0)+\left(\frac{\alpha_{1} A_{r} B^{*}}{\left(1+B^{*}\right)^{2}}-\frac{\alpha_{1} A_{r}}{1+B^{*}}\right) \zeta_{1}(0) \zeta_{2}(0)+\frac{\alpha_{1} A_{r}}{\left(1+B^{*}\right)^{2}} \zeta_{1}^{2}(0) \zeta_{2}(0)-\gamma_{1} \zeta_{1}(0) \zeta_{3}(0) \\
& +\phi_{1} \zeta_{1}(0) \zeta_{4}(0), \\
& Z_{2}=-\frac{r_{1} \zeta_{2}^{2}(0)}{L}-\frac{\alpha_{2} A_{r} I^{*}}{\left(1+B^{*}\right)^{2}} \zeta_{1}^{2}(0)+\left(\frac{\alpha_{2} A_{r}}{1+B^{*}}-\frac{\alpha_{2} A_{r} B^{*}}{\left(1+B^{*}\right)^{2}}\right) \zeta_{1}(0) \zeta_{2}(0)-\frac{\alpha_{2} A_{r} \zeta_{1}^{2}(0) \zeta_{2}(0)}{\left(1+B^{*}\right)^{2}},
\end{aligned}
$$




$$
\begin{gathered}
Z_{3}=-\gamma_{2} \zeta_{1}(0) \zeta_{3}(0), \\
Z_{4}=0
\end{gathered}
$$

For $\zeta=\left(\zeta_{1}, \zeta_{2}, \zeta_{3}, \zeta_{4}\right)^{\mathrm{T}} \in C$.

By the Reisz representation theorem there exists a function $\eta(\Theta, \mu)$ whose components are of bounded variation for $\Theta \in[-1,0]$ such that

$$
L_{\mu} \zeta=\int_{0}^{-1} \mathrm{~d} \eta(\Theta, \mu) \zeta(\Theta)
$$

In view of Equation (29) we can choose

$$
\eta(\Theta, \mu)=\left(\tau_{k}+\mu\right)\left[M_{1} \delta(\Theta)-M_{2} \delta(\Theta+1)\right],
$$

where $\zeta \in C^{1}\left([-1,0], R^{4}\right)$, define

$$
A(\mu) \zeta= \begin{cases}\frac{\mathrm{d} \zeta(\Theta)}{\mathrm{d} \Theta}, & \Theta \in[-1,0), \\ \int_{-1}^{0} \mathrm{~d} \eta(\rho, \mu) \zeta(\rho)=L_{\mu} \zeta, & \Theta=0,\end{cases}
$$

and

$$
R(\mu) \zeta= \begin{cases}0, & \Theta \in[-1,0), \\ f(\zeta, \mu), & \Theta=0 .\end{cases}
$$

The system (28) is the equivalent to

$$
\dot{\chi}_{t}=A(\mu) \chi_{t}+R(\mu) \chi_{t},
$$

where $\chi_{t}(\Theta)=\chi(t+\Theta)$ for $\Theta \in[-1,0]$.

For $\psi \in C^{1}\left([-1,0],\left(R^{4}\right)^{*}\right)$, define

$$
A^{*} \xi(\rho)= \begin{cases}-\frac{\mathrm{d} \xi(\rho)}{\mathrm{d} s}, & \rho \in(0,1], \\ \int_{-1}^{0} \mathrm{~d} \eta^{\mathrm{T}}(t, 0) \xi(-t) & \rho=0 .\end{cases}
$$

and a bilinear inner product

$$
\langle\xi, \zeta\rangle=\bar{\xi}(0) \cdot \zeta(0)-\int_{\Theta=-1}^{0} \int_{v=0}^{\Theta} \bar{\xi}^{\mathrm{T}}(v-\Theta) \mathrm{d} \eta(\Theta) \zeta(v) \mathrm{d} v
$$

where $\eta(\Theta)=\eta(\Theta, 0)$. Then $A(0)$ (from here onwards we shall refer $A(0)$ by $A$ ) and $A^{*}$ are adjoint operators. We know that $\pm i \omega_{0} \tau_{k}$ are the eigenvalues of $A$. Thus, they are also eigenvalues of $A^{*}$. We need to compute eigenvectors of $A$ and $A^{*}$ corresponding to $+i \omega_{0} \tau_{k}$ and $-i \omega_{0} \tau_{k}$ respectively.

Suppose $q(\Theta)=\left(1, a_{1}, a_{2}, a_{3}\right)^{\mathrm{T}} \mathrm{e}^{\mathrm{i} \omega_{0} \tau_{k} \Theta}$ be the eigenvector of $A$ corresponding to eigenvalues $i \omega_{0} \tau_{k}$ then

$$
A q(\Theta)=i \omega_{0} \tau_{k} q(\Theta) \text {, }
$$

which for $\Theta=0$, gives

$$
\tau_{k}\left[\begin{array}{cccc}
i \omega_{0}+B^{*}\left(\frac{r}{K}-\frac{A_{r} \alpha_{1} I^{*}}{\left(1+B^{*}\right)^{2}}\right) & \frac{\alpha_{1} B^{*} A_{r}}{1+B^{*}} & \gamma_{1} B^{*} & -\phi_{1} B^{*} \\
-\frac{\alpha_{2} A_{r} I^{*}}{1+B^{*}}+\frac{\alpha_{2} A_{r} B^{*} I^{*}}{\left(1+B^{*}\right)^{2}} & i \omega_{0}+\frac{r_{1} I^{*}}{L} & 0 & 0 \\
\gamma_{2} T^{*} & Q_{1} & i \omega_{0}+\delta_{0}+\gamma_{2} B^{*} & 0 \\
\phi \mathrm{e}^{-i \omega_{0} \tau_{k}} & 0 & 0 & i \omega_{0}+\phi_{0}
\end{array}\right] q(0)=\left[\begin{array}{l}
0 \\
0 \\
0 \\
0
\end{array}\right] .
$$


Solving the system of Equation (39), we get

$$
a_{1}=\frac{\alpha_{2} A_{r} I^{*} L}{\left(1+B^{*}\right)^{2}\left(i \omega_{0} L+r_{1} I^{*}\right)}, \quad a_{2}=-\frac{\gamma_{2} T^{*}}{i \omega_{0}+\delta_{0}+\gamma_{2} B^{*}}-\frac{\alpha_{2} A_{r} L I^{*} Q_{1}}{\left(1+B^{*}\right)^{2}\left(i \omega_{0} L+r_{1} I^{*}\right)\left(i \omega_{0}+\delta_{0}+\gamma_{2} B^{*}\right)}
$$

and $a_{3}=-\frac{\phi \mathrm{e}^{-i \omega_{0} \tau_{k}}}{i \omega_{0}+\phi_{0}}$.

Similarly calculating $q^{*}(\rho)=\left(1, a_{1}^{*}, a_{2}^{*}, a_{3}^{*}\right)^{\mathrm{T}} \mathrm{e}^{i \omega_{0} \tau_{k} \rho}$ such that

$$
A^{*} q^{*}(\rho)=-i \omega_{0} \tau_{k} q^{*}(\rho),
$$

where

$$
\begin{gathered}
a_{1}^{*}=-\frac{\gamma_{1} B^{*} Q_{1}}{\left(-i \omega_{0}+\frac{r_{1} I^{*}}{L}\right)\left(i \omega_{0}-\delta_{0}-\gamma_{2} B^{*}\right)}-\frac{\alpha_{1} B^{*} A_{r}}{\left(1+B^{*}\right)\left(-i \omega_{0}+\frac{r_{1} I^{*}}{L}\right)}, a_{2}^{*}=\frac{\gamma_{1} B^{*}}{i \omega_{0}-\delta_{0}-\gamma_{2} B^{*}} \\
a_{3}^{*}=-\frac{\phi_{1} B^{*}}{\phi_{0}-i \omega_{0}} .
\end{gathered}
$$

Now the normalization condition gives

$$
\begin{gathered}
\left\langle q^{*}(\rho) \cdot q(\Theta)\right\rangle=1, \\
\bar{q}^{*}(0) \cdot q(0)-\bar{D} \int_{\Theta=-1}^{0} \int_{\nu=0}^{\Theta} \bar{q}^{*}(0) \mathrm{e}^{-i \omega_{0} \tau_{k}(v-\Theta)} \mathrm{d} \eta(\Theta) \times q(0) \mathrm{e}^{i \omega_{0} \tau_{k} v} \mathrm{~d} v=1 \\
\bar{D}\left[1+a_{1} \bar{a}_{1}^{*}+a_{2} \bar{a}_{2}^{*}+a_{3} \bar{a}_{3}^{*}-\mathrm{e}^{i \omega_{0} \tau_{k}} \tau_{k} \phi \bar{a}_{3}^{*}\right]=1 .
\end{gathered}
$$

Thus, $\bar{D}$ is so chosen such that

$$
\bar{D}=\frac{1}{1+a_{1} \bar{a}_{1}^{*}+a_{2} \bar{a}_{2}^{*}+a_{3} \bar{a}_{3}^{*}-\mathrm{e}^{i \omega_{0} \tau_{k}} \tau_{k} \phi \bar{a}_{3}^{*}} .
$$

Proceeding same as [10] and using same notation, we compute the coordinates to describe the center manifold $C_{0}$ at $\mu=0$. Let $\chi_{t}$ be solution of Equation (35) when $\mu=0$. Define

$$
Z(t)=\left\langle q^{*}, \chi_{t}\right\rangle, \quad W(t, \Theta)=\chi_{t}(\Theta)-2 \operatorname{Re}\{Z(t) q(\Theta)\} .
$$

On the center manifold $C_{0}$, we have

$$
\begin{gathered}
W(t, \Theta)=W(Z, \bar{Z}, \Theta) \\
W(z, \bar{z}, \Theta)=W_{20}(\Theta) \frac{z^{2}}{2}+W_{11}(\Theta) z \bar{Z}+W_{02}(\Theta) \frac{\bar{z}^{2}}{2}+\cdots .
\end{gathered}
$$

$Z$ and $\bar{Z}$ are local coordinates for center manifold $C_{0}$ in the direction of $q^{*}$ and $\bar{q}^{*}$. Note that $W$ is real if $\chi_{t}$ is real. We only consider real solutions. For solution $\chi_{t} \in C_{0}$ of Equation (35). Since $\mu=0$, we have

$$
\begin{aligned}
\dot{z} & =i \omega_{0} \tau_{k} z+\bar{q}^{*}(0) \cdot f(0, W(z, \bar{z}, 0)+2 \operatorname{Re}\{z q(0)\}) \\
& =i \omega_{0} \tau_{k} z+\bar{q}^{*}(0) \cdot f_{0}(z, \bar{z}) .
\end{aligned}
$$

We rewrite this equation as

$$
\dot{z}=i \omega_{0} \tau_{k} z+g(z, \bar{z}),
$$

where 


$$
g(z, \bar{z})=\bar{q}^{*}(0) \cdot f_{0}(z, \bar{z})=g_{20} \frac{z^{2}}{2}+g_{11} z \bar{Z}+g_{02} \frac{\bar{z}^{2}}{2}+g_{21} \frac{z^{2} \bar{Z}}{2}+\cdots
$$

It follows from (42) and (44) that

$$
\begin{gathered}
\chi_{t}(\Theta)=W(z, \bar{z}, \Theta)+2 \operatorname{Re}\{z q(\Theta)\}, \\
=W_{20}(\Theta) \frac{z^{2}}{2}+W_{11}(\Theta) z \bar{z}+W_{02}(\Theta) \frac{\bar{z}^{2}}{2}+z\left(1, a_{1}, a_{2}, a_{3}\right)^{\mathrm{T}} \mathrm{e}^{i \omega_{0} \tau_{k} \Theta}+\bar{z}\left(1, \bar{a}_{1}, \bar{a}_{2}, \bar{a}_{3}\right)^{\mathrm{T}} \mathrm{e}^{-i \omega_{0} \tau_{k} \Theta}+\cdots .
\end{gathered}
$$

Also we have

$$
g(z, \bar{z})=\bar{q}^{*}(0) \cdot f_{0}\left(0, \chi_{t}\right)=\tau_{k} \bar{D}\left(1, \bar{a}_{1}^{*}, \bar{a}_{2}^{*}, \bar{a}_{3}^{*}\right)^{\mathrm{T}}\left(\begin{array}{c}
V_{1} \\
V_{2} \\
V_{3} \\
0
\end{array}\right)
$$

where

$$
\begin{gathered}
V_{1}=-\left(\frac{r}{K}-\frac{\alpha_{1} A_{r} I^{*}}{\left(1+B^{*}\right)^{2}}\right) \chi_{1 t}^{2}(0)+\left\{\frac{\alpha_{1} A_{r} B^{*}}{\left(1+B^{*}\right)^{2}}-\frac{\alpha_{1} A_{r}}{1+B^{*}}\right\} \chi_{1 t}(0) \chi_{2 t}(0)+\frac{\alpha_{1} A_{r}}{\left(1+B^{*}\right)^{2}} \chi_{1 t}^{2}(0) \chi_{2 t}(0) \\
-\gamma_{1} \chi_{1 t}(0) \chi_{3 t}(0)+\phi_{1} \chi_{1 t}(0) \chi_{4 t}(0), \\
V_{2}=-\frac{r_{1}}{L} \chi_{2 t}^{2}(0)-\frac{\alpha_{2} A_{r} I^{*}}{\left(1+B^{*}\right)^{2}} \chi_{1 t}^{2}(0)+\left\{\frac{\alpha_{2} A_{r}}{1+B^{*}}-\frac{\alpha_{2} A_{r} B^{*}}{\left(1+B^{*}\right)^{2}}\right\} \chi_{1 t}(0) \chi_{2 t}(0)-\frac{\alpha_{2} A_{r}}{\left(1+B^{*}\right)^{2}} \chi_{1 t}^{2}(0) \chi_{2 t}(0) \\
V_{3}=-\gamma_{2} \chi_{1 t}(0) \chi_{3 t}(0) .
\end{gathered}
$$

So that

$$
\begin{gathered}
\chi_{1 t}(\Theta)=W_{20}^{(1)}(\Theta) \frac{z^{2}}{2}+W_{11}^{(1)}(\Theta) z \bar{Z}+W_{02}^{(1)}(\Theta) \frac{\bar{z}^{2}}{2}+z \mathrm{e}^{i \omega_{0} \tau_{k} \Theta}+\bar{z}^{-i \omega_{0} \tau_{k} \Theta}+\cdots \\
\chi_{2 t}(\Theta)=W_{20}^{(2)}(\Theta) \frac{z^{2}}{2}+W_{11}^{(2)}(\Theta) z \bar{Z}+W_{02}^{(2)}(\Theta) \frac{\bar{z}^{2}}{2}+a_{1} z \mathrm{z}^{i \omega_{0} \tau_{k} \Theta}+\bar{a}_{1} \bar{z} \mathrm{e}^{-i \omega_{0} \tau_{k} \Theta}+\cdots \\
\chi_{3 t}(\Theta)=W_{20}^{(3)}(\Theta) \frac{z^{2}}{2}+W_{11}^{(3)}(\Theta) z \bar{Z}+W_{02}^{(3)}(\Theta) \frac{\bar{Z}^{2}}{2}+a_{2} z \mathrm{z}^{i \omega_{0} \tau_{k} \Theta}+\bar{a}_{2} \bar{z} \mathrm{e}^{-i \omega_{0} \tau_{k} \Theta}+\cdots \\
\chi_{4 t}(\Theta)=W_{20}^{(4)}(\Theta) \frac{z^{2}}{2}+W_{11}^{(4)}(\Theta) z \bar{z}+W_{02}^{(4)}(\Theta) \frac{\bar{Z}^{2}}{2}+a_{3} z \mathrm{e}^{i \omega_{0} \tau_{k} \Theta}+\bar{a}_{3} \bar{z} \mathrm{e}^{-i \omega_{0} \tau_{k} \Theta}+\cdots
\end{gathered}
$$

Thus

$$
\begin{gathered}
\chi_{1 t}(0)=z+\bar{z}+W_{20}^{(1)}(0) \frac{z^{2}}{2}+W_{11}^{(1)}(0) z \bar{Z}+W_{02}^{(1)}(0) \frac{\bar{z}^{2}}{2}+\cdots \\
\chi_{2 t}(0)=a_{1} z+\bar{a}_{1} \bar{z}+W_{20}^{(2)}(0) \frac{z^{2}}{2}+W_{11}^{(2)}(0) z \bar{z}+W_{02}^{(2)}(0) \frac{\bar{z}^{2}}{2}+\cdots \\
\chi_{3 t}(0)=a_{2} z+\bar{a}_{2} \bar{z}+W_{20}^{(3)}(0) \frac{z^{2}}{2}+W_{11}^{(3)}(0) z \bar{z}+W_{02}^{(3)}(0) \frac{\bar{z}^{2}}{2}+\cdots \\
\chi_{4 t}(0)=a_{3} z+\bar{a}_{3} \bar{z}+W_{20}^{(4)}(0) \frac{z^{2}}{2}+W_{11}^{(4)}(0) z \bar{z}+W_{02}^{(4)}(0) \frac{\bar{z}^{2}}{2}+\cdots
\end{gathered}
$$

Now 


$$
\begin{aligned}
g(z, \bar{z}) & =\tau_{k} \bar{D}\left(1, \bar{a}_{1}^{*}, \bar{a}_{2}^{*}, \bar{a}_{3}^{*}\right)^{\mathrm{T}} \cdot\left(\begin{array}{c}
V_{1} \\
V_{2} \\
V_{3} \\
0
\end{array}\right), \\
& =\tau_{k} \bar{D}\left[-\left(\frac{r}{K}-\frac{\alpha_{1} A_{r} I^{*}}{\left(1+B^{*}\right)^{2}}\right)-\frac{\bar{a}_{1}^{*} \alpha_{2} A_{r} I^{*}}{\left(1+B^{*}\right)^{2}}\right] \chi_{1 t}^{2}(0) \\
& +\tau_{k} \bar{D}\left[\left\{\frac{\alpha_{1} A_{r} B^{*}}{\left(1+B^{*}\right)^{2}}-\frac{\alpha_{1} A_{r}}{1+B^{*}}\right\}+\bar{a}_{1}^{*}\left\{\frac{\alpha_{2} A_{r}}{1+B^{*}}-\frac{\alpha_{2} A_{r} B^{*}}{\left(1+B^{*}\right)^{2}}\right\}\right] \cdot \chi_{1 t}(0) \chi_{2 t}(0) \\
& +\tau_{k} \bar{D}\left[\frac{\alpha_{1} A_{r}}{\left(1+B^{*}\right)^{2}}-\frac{\bar{a}_{1}^{*} \alpha_{2} A_{r}}{\left(1+B^{*}\right)^{2}}\right] \chi_{1 t}^{2}(0) \chi_{2 t}(0) \\
& -\tau_{k} \bar{D}\left\{\gamma_{1}+\bar{a}_{2}^{*} \gamma_{2}\right\} \chi_{1 t}(0) \chi_{3 t}(0) \\
& +\tau_{k} \bar{D} \phi_{1} \chi_{1 t}(0) \chi_{4 t}(0) \\
& -\tau_{k} \bar{D} \bar{a}_{1}^{*} \frac{r_{1}}{L} \chi_{2 t}^{2}(0) .
\end{aligned}
$$

Comparing the coefficients in (37) with those in (50), we get

$$
\begin{aligned}
& g_{20}= 2 \tau_{k} \bar{D}\left[-\left(\frac{r}{K}-\frac{\alpha_{1} A_{r} I^{*}}{\left(1+B^{*}\right)^{2}}+\frac{\bar{a}_{1}^{*} \alpha_{2} A_{r} I^{*}}{\left(1+B^{*}\right)^{2}}\right)+a_{1}\left\{\frac{\alpha_{1} A_{r} B^{*}}{\left(1+B^{*}\right)^{2}}-\frac{\alpha_{1} A_{r}}{1+B^{*}}\right\}\right. \\
&\left.+a_{1} \bar{a}_{1}^{*}\left\{\frac{\alpha_{2} A_{r}}{1+B^{*}}-\frac{\alpha_{2} A_{r} B^{*}}{\left(1+B^{*}\right)^{2}}\right\}+a_{2}\left\{-\gamma_{1}-\bar{a}_{2}^{*} \gamma_{2}\right\}+a_{3} \phi_{1}-\bar{a}_{1}^{*} \frac{r_{1}}{L} a_{1}^{2}\right], \\
& g_{11}=2 \tau_{k} \bar{D}\left[-\left\{\frac{r}{K}-\frac{\alpha_{1} A_{r} I^{*}}{\left(1+B^{*}\right)^{2}}+\frac{\bar{a}_{1}^{*} \alpha_{2} A_{r} I^{*}}{\left(1+B^{*}\right)^{2}}\right\}+\operatorname{Re}\left(a_{1}\right)\left\{\frac{\alpha_{1} A_{r} B^{*}}{\left(1+B^{*}\right)^{2}}-\frac{\alpha_{1} A_{r}}{1+B^{*}}\right\}\right. \\
&+\bar{a}_{1}^{*}\left\{\frac{\alpha_{2} A_{r}}{\left.1+B^{*}-\frac{\alpha_{2} A_{r} B^{*}}{\left(1+B^{*}\right)^{2}}\right\} \operatorname{Re}\left(a_{1}\right)}\right. \\
&-\left.\operatorname{Re}\left(a_{2}\right)\left\{\gamma_{1}+\bar{a}_{2}^{*} \gamma_{2}\right\}+\phi_{1} \operatorname{Re}\left(a_{3}\right)-\frac{\bar{a}_{1}^{*} r_{1}}{L}\left|a_{1}\right|^{2}\right], \\
& g_{02}= 2 \tau_{k} \bar{D}\left[-\left(\frac{r}{K}-\frac{\alpha_{1} A_{r} I^{*}}{\left(1+B^{*}\right)^{2}}+\frac{\bar{a}_{1}^{*} \alpha_{2} A_{r} I^{*}}{\left(1+B^{*}\right)^{2}}\right)+\bar{a}_{1}\left\{\frac{\alpha_{1} A_{r} B^{*}}{\left(1+B^{*}\right)^{2}}-\frac{\alpha_{1} A_{r}}{1+B^{*}}\right\}\right. \\
&\left.+\bar{a}_{1} \bar{a}_{1}^{*}\left\{\frac{\alpha_{2} A_{r}}{1+B^{*}}-\frac{\alpha_{2} A_{r} B^{*}}{\left(1+B^{*}\right)^{2}}\right\}-\bar{a}_{2}\left\{-\gamma_{1}-\bar{a}_{2}^{*} \gamma_{2}\right\}+\bar{a}_{3} \phi_{1}-\bar{a}_{1}^{*} \frac{r_{1}}{L} \bar{a}_{1}^{2}\right],
\end{aligned}
$$




$$
\begin{aligned}
g_{21}= & 2 \tau_{k} \bar{D}\left[-\left(\frac{r}{K}-\frac{\alpha_{1} A_{r} I^{*}}{\left(1+B^{*}\right)^{2}}+\frac{\bar{a}_{1}^{*} \alpha_{2} A_{r} I^{*}}{\left(1+B^{*}\right)^{2}}\right)\left(W_{20}^{(1)}(0)+2 W_{11}^{(1)}(0)\right)\right. \\
& +\left\{\frac{\alpha_{1} A_{r} B^{*}}{\left(1+B^{*}\right)^{2}}-\frac{\alpha_{1} A_{r}}{1+B^{*}}+\frac{\alpha_{2} A_{r} \bar{a}_{1}^{*}}{1+B^{*}}-\frac{\alpha_{2} A_{r} B^{*} \bar{a}_{1}^{*}}{\left(1+B^{*}\right)^{2}}\right\}\left(a_{1} W_{11}^{(1)}(0)+\bar{a}_{1} \frac{W_{20}^{(1)}(0)}{2}+\frac{W_{20}^{(2)}(0)}{2}+W_{11}^{(2)}(0)\right) \\
& +\left\{\frac{\alpha_{1} A_{r}}{\left(1+B^{*}\right)^{2}}-\frac{\bar{a}_{1}^{*} \alpha_{2} A_{r}}{\left(1+B^{*}\right)^{2}}\right\}\left(2 a_{1}+\bar{a}_{1}\right)-\left(\gamma_{1}+\bar{a}_{2}^{*} \gamma_{2}\right)\left(a_{2} W_{11}^{(1)}(0)+\frac{\bar{a}_{2}}{2} W_{20}^{(1)}(0)+\frac{W_{20}^{(3)}(0)}{2}+W_{11}^{(3)}(0)\right) \\
& +\phi_{1}\left\{a_{3} W_{11}^{(1)}(0)+\frac{\bar{a}_{3}}{2} W_{20}^{(1)}(0)+\frac{W_{20}^{(4)}(0)}{2}+W_{11}^{(4)}(0)\right\}-\frac{\bar{a}_{1}^{*} r_{1}}{L}\left\{\bar{a}_{1} W_{20}^{(2)}(0)+2 a_{1} W_{11}^{(2)}(0)\right\} .
\end{aligned}
$$

In order to compute $g_{21}$, we need to $W_{20}(\Theta)$ and $W_{11}(\Theta)$. From Equations (42) and (45) we have

$$
\begin{gathered}
\dot{W}=\dot{\chi}_{t}-\dot{z} q-\dot{z} \bar{q} \\
= \begin{cases}A W-2 \operatorname{Re}\left\{\bar{q}^{*}(0) \cdot f_{0} q(\Theta)\right\}, & \Theta \in[-1,0), \\
A W-2 \operatorname{Re}\left\{\bar{q}^{*}(0) \cdot f_{0} q(0)\right\}+f_{0}, & \Theta=0,\end{cases} \\
=A W+H(z, \bar{z}, \Theta)
\end{gathered}
$$

with

$$
H(z, \bar{Z}, \Theta)=H_{20}(\Theta) \frac{z^{2}}{2}+H_{11}(\Theta) z \bar{Z}+H_{02}(\Theta) \frac{\bar{Z}^{2}}{2}+\cdots .
$$

Also, on $C_{0}$, using chain rule, we get

$$
\dot{W}=W_{z} \dot{z}+W_{\bar{z}} \dot{\bar{z}}
$$

It follows from (46), (52) and (54)

$$
\begin{gathered}
\left(A-2 i \omega_{0} \tau_{k}\right) W_{20}=-H_{20}, \\
A W_{11}=-H_{11}
\end{gathered}
$$

etc. Now for $\Theta \in[-1,0)$

$$
\begin{aligned}
H(z, \bar{z}, \Theta) & =-\bar{q}^{*}(0) \cdot f_{0} q(\Theta)-\bar{q}^{*}(0) \cdot \bar{f}_{0} \bar{q}(\Theta) \\
& =-g(z, \bar{z}) q(\Theta)-\bar{g}(z, \bar{z}) \bar{q}(\Theta) \\
& =-\left(g_{20} q(\Theta)+\bar{g}_{02} \bar{q}(\Theta)\right) \frac{z^{2}}{2}-\left(g_{11} q(\Theta)+\bar{g}_{11} \bar{q}(\Theta)\right) z \bar{z}+\cdots,
\end{aligned}
$$

which on comparing the coefficients with (53) gives

$$
\begin{gathered}
H_{20}(\Theta)=-g_{20} q(\Theta)-\bar{g}_{02} \bar{q}(\Theta), \\
H_{11}(\Theta)=-g_{11} q(\Theta)-\bar{g}_{11} \bar{q}(\Theta) .
\end{gathered}
$$

From (56), (58) and the definition of $A$, we have

$$
W_{20}^{\prime}(\Theta)=2 i \omega_{0} \tau_{k} W_{20}(\Theta)+g_{20} q(\Theta)+\bar{g}_{02} \bar{q}(\Theta) .
$$

Note that $q(\Theta)=q(0) \mathrm{e}^{i \omega_{0} \tau_{k} \Theta}$, hence

$$
W_{20}(\Theta)=\frac{i g_{20}}{\omega_{0} \tau_{k}} q(\Theta)+\frac{i \bar{g}_{20}}{3 \omega_{0} \tau_{k}} \bar{q}(\Theta)+F_{1} \mathrm{e}^{2 i \omega_{0} \tau_{k} \Theta} .
$$


Similarly from (56), (59) and the definition of $A$, we have

$$
\begin{gathered}
W_{11}^{\prime}(\Theta)=g_{11} q(\Theta)+\bar{g}_{11} \bar{q}(\Theta) \\
W_{11}(\Theta)=-\frac{i g_{11}}{\omega_{0} \tau_{k}} q(\Theta)+\frac{i \bar{g}_{11}}{\omega_{0} \tau_{k}} \bar{q}(\Theta)+F_{2}
\end{gathered}
$$

where $F_{1}=\left(F_{1}^{(1)}, F_{1}^{(2)}, F_{1}^{(3)}, F_{1}^{(4)}\right)$ and $F_{2}=\left(F_{2}^{(1)}, F_{2}^{(2)}, F_{2}^{(3)}, F_{2}^{(4)}\right) \in R^{4}$ are constant vectors, to be determined.

It follows from the definition of $A$ and (45) that

$$
\begin{gathered}
\int_{-1}^{0} \mathrm{~d} \eta(\Theta) W_{20}(\Theta)=2 i \omega_{0} \tau_{k} W_{20}(0)-H_{20}(0) \\
\int_{-1}^{0} \mathrm{~d} \eta(\Theta) W_{11}(\Theta)=-H_{11}(0) .
\end{gathered}
$$

From Equations (61) and (63) we get

$$
H_{20}(0)=-g_{20} q(0)-\bar{g}_{02} \bar{q}(0)+2 \tau_{k}\left(\begin{array}{c}
-\left(\frac{r}{K}-\frac{\alpha_{1} A_{r} I^{*}}{\left(1+B^{*}\right)^{2}}-\frac{\alpha_{1} a_{1} A_{r} B^{*}}{\left(1+B^{*}\right)^{2}}+\frac{\alpha_{1} a_{1} A_{r}}{1+B^{*}}+a_{2} \gamma_{1}-a_{3} \phi_{1}\right) \\
-\frac{\alpha_{2} A_{r} I^{*}}{\left(1+B^{*}\right)^{2}}+a_{1}\left(\frac{\alpha_{2} A_{r}}{1+B^{*}}-\frac{\alpha_{2} A_{r} B^{*}}{\left(1+B^{*}\right)^{2}}\right)-\frac{r_{1} a_{1}^{2}}{L} \\
-a_{2} \gamma_{2} \\
0
\end{array}\right),
$$

and

$$
=-g_{11} q(0)-\bar{g}_{11} \bar{q}(0)+2 \tau_{k}\left(\begin{array}{c}
H_{11}(0) \\
-\left(\frac{r}{K}-\frac{\alpha_{1} A_{r} I^{*}}{\left(1+B^{*}\right)^{2}}-\left(\frac{\alpha_{1} A_{r} B^{*}}{\left(1+B^{*}\right)^{2}}-\frac{\alpha_{1} A_{r}}{1+B^{*}}\right) \operatorname{Re}\left(a_{1}\right)+\operatorname{Re}\left(a_{2}\right) \gamma_{1}-\operatorname{Re}\left(a_{3}\right) \phi_{1}\right) \\
-\frac{\alpha_{2} A_{r} I^{*}}{\left(1+B^{*}\right)^{2}}+\operatorname{Re}\left(a_{1}\right)\left(\frac{\alpha_{2} A_{r}}{1+B^{*}}-\frac{\alpha_{2} A_{r} B^{*}}{\left(1+B^{*}\right)^{2}}\right)-\frac{r_{1}\left|a_{1}\right|^{2}}{L} \\
-\operatorname{Re}\left(a_{2}\right) \gamma_{2} \\
0
\end{array}\right) .
$$

Using (61) and (66) in (64) and noting that $q(\Theta)$ is eigenvector of $A$, we have

$$
\left(2 i \omega_{0} \tau_{k} I-\int_{1}^{0} \mathrm{e}^{2 i \omega_{0} \tau_{k} \Theta} \mathrm{d} \eta(\Theta)\right) F_{1}=2 \tau_{k}\left(\begin{array}{c}
-\left(\frac{r}{K}-\frac{\alpha_{1} A_{r} I^{*}}{\left(1+B^{*}\right)^{2}}-\frac{\alpha_{1} a_{1} A_{r} B^{*}}{\left(1+B^{*}\right)^{2}}+\frac{\alpha_{1} a_{1} A_{r}}{1+B^{*}}+a_{2} \gamma_{1}-a \phi_{1}\right) \\
-\frac{\alpha_{2} A_{r} I^{*}}{\left(1+B^{*}\right)^{2}}+a_{1}\left(\frac{\alpha_{2} A_{r}}{1+B^{*}}-\frac{\alpha_{2} A_{r} B^{*}}{\left(1+B^{*}\right)^{2}}\right)-\frac{r_{1} a_{1}^{2}}{L} \\
-a_{2} \gamma_{2} \\
0
\end{array}\right)
$$

i.e. 


$$
\begin{aligned}
& \left(\begin{array}{cccc}
2 i \omega_{0}+B^{*}\left(\frac{r}{K}-\frac{A_{r} \alpha_{1} I^{*}}{\left(1+B^{*}\right)^{2}}\right) & \frac{\alpha_{1} B^{*} A_{r}}{1+B^{*}} & \gamma_{1} B^{*} & -\phi_{1} B^{*} \\
-\frac{\alpha_{2} A_{r} I^{*}}{1+B^{*}}+\frac{\alpha_{2} A_{r} B^{*} I^{*}}{\left(1+B^{*}\right)^{2}} & 2 i \omega_{0}+\frac{r_{1} I^{*}}{L} & 0 & 0 \\
\gamma_{2} T^{*} & Q_{1} & 2 i \omega_{0}+\delta_{0}+\gamma_{2} B^{*} & 0 \\
\phi \mathrm{e}^{-2 i \omega_{0} \tau_{k}} & 0 & 0 & 2 i \omega_{0}+\phi_{0}
\end{array}\right)\left(\begin{array}{c}
F_{1}^{(1)} \\
F_{1}^{(2)} \\
F_{1}^{(3)} \\
F_{1}^{(4)}
\end{array}\right) \\
& =2\left(\begin{array}{c}
-\left(\frac{r}{K}-\frac{\alpha_{1} A_{r} I^{*}}{\left(1+B^{*}\right)^{2}}-\frac{\alpha_{1} a_{1} A_{r} B^{*}}{\left(1+B^{*}\right)^{2}}+\frac{\alpha_{1} a_{1} A_{r}}{1+B^{*}}+a_{2} \gamma_{1}-a_{3} \phi_{1}\right) \\
-\frac{\alpha_{2} A_{r} I^{*}}{\left(1+B^{*}\right)^{2}}+a_{1}\left(\frac{\alpha_{2} A_{r}}{1+B^{*}}-\frac{\alpha_{2} A_{r} B^{*}}{\left(1+B^{*}\right)^{2}}\right)-\frac{r_{1} a_{1}^{2}}{L} \\
-a_{2} \gamma_{2} \\
0
\end{array}\right) .
\end{aligned}
$$

Similarly using (63) and (67) in (65), we get

$$
\begin{aligned}
& \left(\begin{array}{cccc}
B^{*}\left(\frac{r}{K}-\frac{A_{r} \alpha_{1} I^{*}}{\left(1+B^{*}\right)^{2}}\right) & \frac{\alpha_{1} B^{*} A_{r}}{1+B^{*}} & \gamma_{1} B^{*} & -\phi_{1} B^{*} \\
-\frac{\alpha_{2} A_{r} I^{*}}{1+B^{*}}+\frac{\alpha_{2} A_{r} B^{*} I^{*}}{\left(1+B^{*}\right)^{2}} & \frac{r_{1} I^{*}}{L} & 0 & 0 \\
\gamma_{2} T^{*} & Q_{1} & \delta_{0}+\gamma_{2} B^{*} & 0 \\
\phi & 0 & 0 & \phi_{0}
\end{array}\right)\left(\begin{array}{c}
F_{2}^{(1)} \\
F_{2}^{(2)} \\
F_{2}^{(3)} \\
F_{2}^{(4)}
\end{array}\right) \\
& =2\left(\begin{array}{c}
-\left(\frac{r}{K}-\frac{\alpha_{1} A_{r} I^{*}}{\left(1+B^{*}\right)^{2}}-\left(\frac{\alpha_{1} A_{r} B^{*}}{\left(1+B^{*}\right)^{2}}-\frac{\alpha_{1} A_{r}}{1+B^{*}}\right) \operatorname{Re}\left(a_{1}\right)+\operatorname{Re}\left(a_{2}\right) \gamma_{1}-\operatorname{Re}\left(a_{3}\right) \phi_{1}\right) \\
-\frac{\alpha_{2} A_{r} I^{*}}{\left(1+B^{*}\right)^{2}}+\operatorname{Re}\left(a_{1}\right)\left(\frac{\alpha_{2} A_{r}}{1+B^{*}}-\frac{\alpha_{2} A_{r} B^{*}}{\left(1+B^{*}\right)^{2}}\right)-\frac{r_{1}\left|a_{1}\right|^{2}}{L} \\
-\operatorname{Re}\left(a_{2}\right) \gamma_{2} \\
0
\end{array}\right) .
\end{aligned}
$$

We solve system (69) for $F_{1}$ and (70) for $F_{2}$ and using these values are determine $W_{20}$ and $W_{11}$ and hence $g_{21}$. Now to determine the direction, stability and period of bifurcating periodic solutions from critical point at the critical value $\tau=\tau_{k}$ we can compute the following necessary quantities as given by [10].

$$
\begin{gathered}
C_{1}(0)=\frac{i}{2 \omega_{0} \tau_{k}}\left(g_{11} g_{20}-2\left|g_{11}\right|^{2}-\frac{\left|g_{02}\right|^{2}}{3}\right)+\frac{g_{21}}{2} \\
\mu_{2}=-\frac{\operatorname{Re}\left\{c_{1}(0)\right\}}{\operatorname{Re}\left\{\psi^{\prime}\left(\tau_{k}\right)\right\}} \\
b_{2}=2 \operatorname{Re}\left\{c_{1}(0)\right\}
\end{gathered}
$$




$$
T_{2}=-\frac{I_{m}\left\{c_{1}(0)\right\}+\mu I_{m}\left\{\psi^{\prime}\left(\tau_{k}\right)\right\}}{\omega_{0} \tau_{k}} .
$$

Hence, using the results of [10]. We have the following theorem.

Theorem (3.2.1): If $\mu_{2}>0\left(\mu_{2}<0\right)$, then the Hopf bifurcation is supercritical (subcritical) and the bifurcating periodic solutions exist for $\tau>\tau_{k}\left(\tau<\tau_{k}\right)$. The bifurcating periodic solution is stable (unstable) if $b_{2}<0\left(b_{2}>0\right)$ and the period increase (decrease) if $T_{2}>0\left(T_{2}<0\right)$.

\section{Numerical Support}

In this section, we present numerical simulation to illustrate the results obtained in the previous sections. The system (4) is solved using the MATLAB software package under the following set of parameters.

(a) $r=2, K=100, \alpha_{1}=0.2, A_{r}=0.6, \gamma_{1}=0.01, L=100, \alpha_{2}=0.1, Q_{0}=1, Q_{1}=0.2, \delta_{0}=0.5$,

$$
\gamma_{2}=0.01, r_{1}=1, \phi=0.01, \phi_{0}=0.02, \phi_{1}=0.8 \text {. }
$$

The interior equilibrium point of system (4) with data (a) is

$$
B^{*}=99.1014, \quad I^{*}=145.94, \quad T^{*}=20.2466, \quad T_{c}^{*}=0.449306 .
$$

Then, we can easily obtain that $\left(\mathrm{H}_{1}\right)$ to be satisfied. By computation, we have $\omega=0.395806, \tau_{0}=5.82023$. The transversality condition (27) is satisfied as $\left[\frac{\mathrm{d}(\operatorname{Re} \psi)}{\mathrm{d} \tau}\right]_{\tau=\tau_{0}}=1.20963 \neq 0$.

The stability behavior of the system (4) for $\tau=0$ can be depicted by Figure 1. To check the dynamic behavior of the system (4) for $\tau \neq 0$ can be seen by Figure 2 and Figure 3. A Hopf bifurcation occurs at $E_{3}\left(B^{*}, I^{*}, T^{*}, T_{c}^{*}\right)$ when $\tau=\tau_{0}=5.82023$ and small amplitude periodic solution around $E_{3}\left(B^{*}, I^{*}, T^{*}, T_{c}^{*}\right)$ and this can be visualized from Figure 2 and Figure 3. From Figure 2 and Figure 3 we can see that when $\tau=1<\tau_{0}=5.82023$ the system is stable and for $\tau=8>\tau_{0}=5.82023$ the system becomes unstable. An alternative resource has a strong impact on the depletion of forestry biomass which can be seen from Figure 4. From this we can see increasing the value of alternative resource the concentration of forestry biomass increases and also controlling the instability of the system (4) when $\tau=8>\tau_{0}=5.82023$ see Figure 4(b). Now see impact of other factors on forestry biomass. Increasing the value of $\alpha_{1}$ the concentration of forest biomass increases

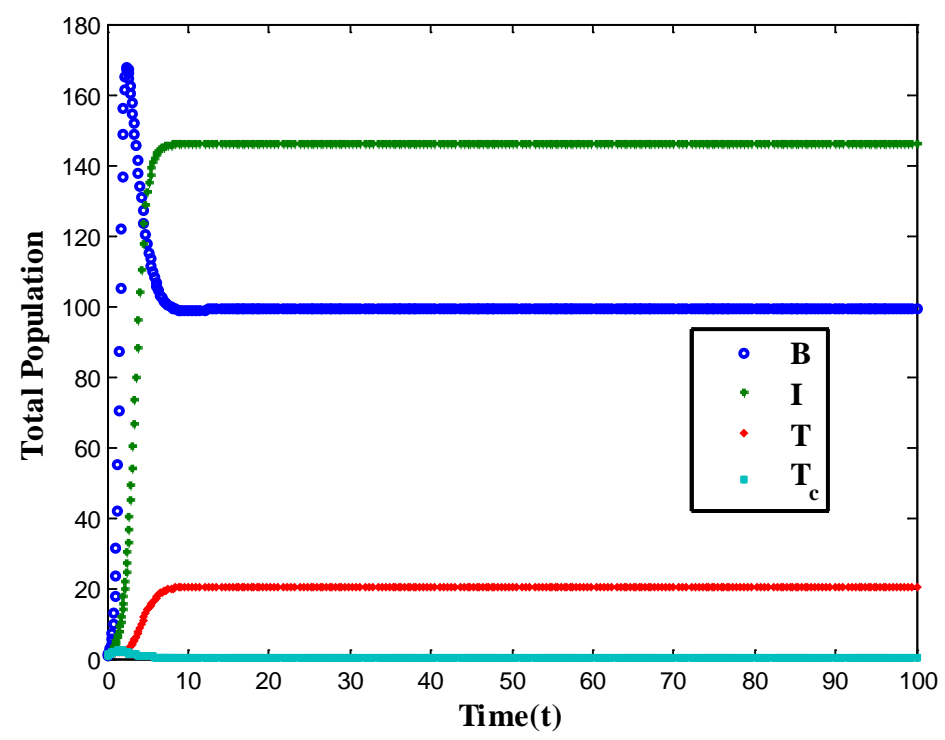

Figure 1. Stable behavior of $B, I, T$ and $T_{c}$ with time, when $\tau=0$ and other parameter values are same as (a). 


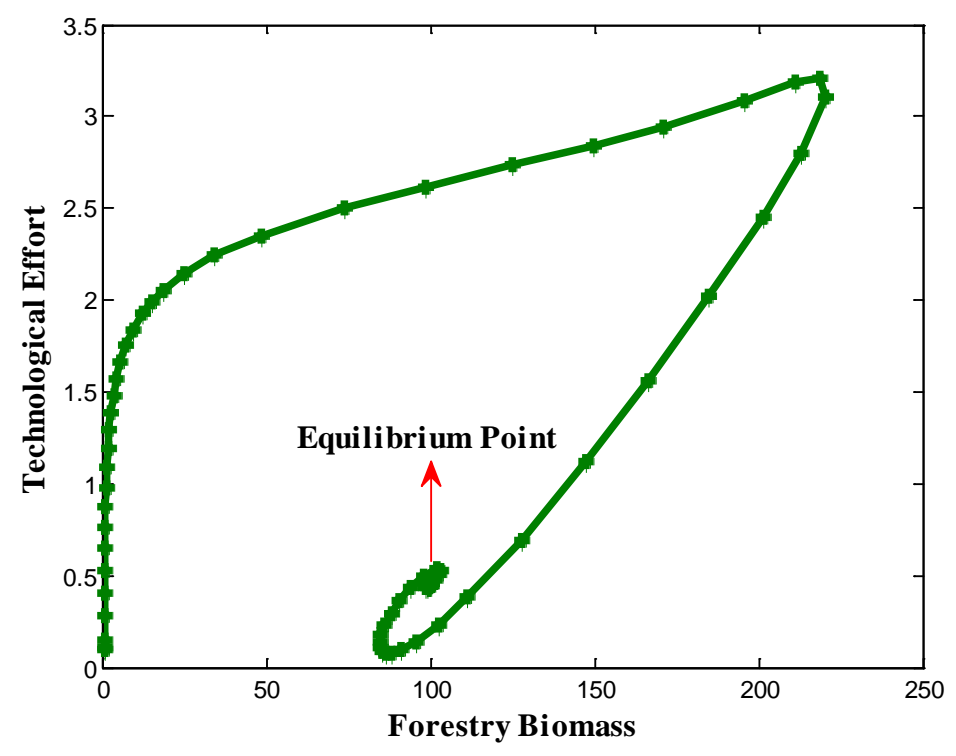

(a)

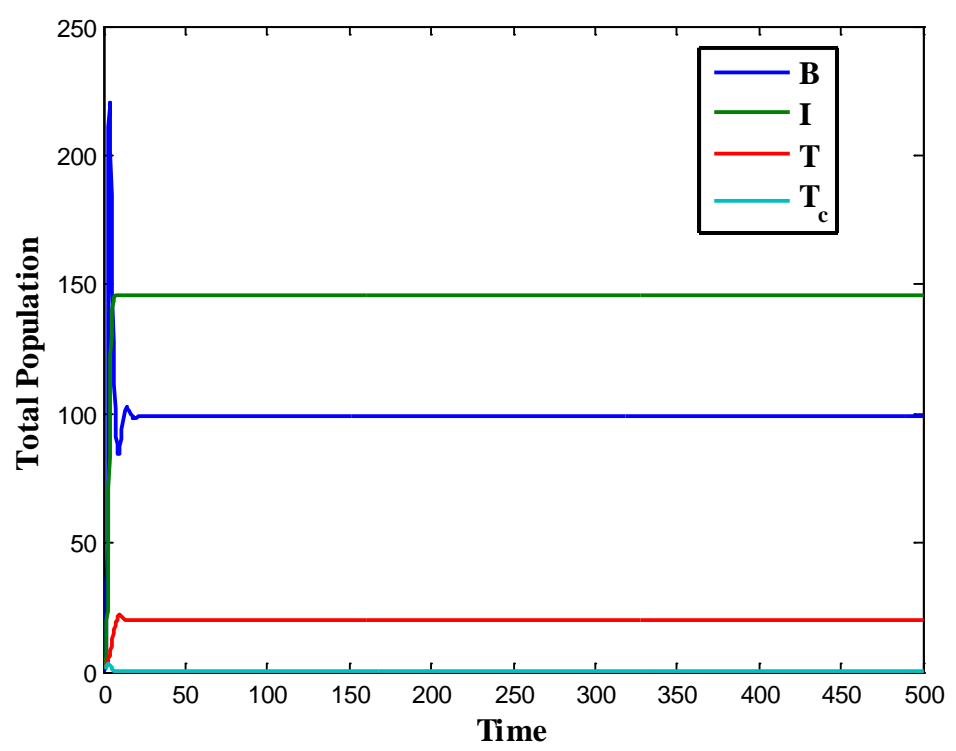

(b)

Figure 2. Trajectory portrait and phase portrait of system (4) for $\tau=1<\tau_{0}=5.82023$ and other parameters are same as (a).

refer Figure 5. From Figure 6 we see that the concentration of forestry biomass decreases when the value of $\gamma_{1}$ increases. Increasing the values of $\phi_{1}$ and $\phi$ the concentration of forestry biomass increases refer Figure 7 and Figure 8.

Now to verify the result of Theorem (3.2.1), we have shown the variation of variables $B, I, T$ and $T_{c}$ for $\tau=1$ and $\tau=8$ in Figure 2 and Figure 3 respectively. Also for the above set of parameter values, we get $c_{1}(0)=-0.19028-15.1071 \mathrm{i}, \mu_{2}=0.157305, b_{2}=-0.38056$ and $T_{2}=6.00118$. Since $\mu_{2}>0$, the Hopf bifurcation is supercritical and the direction of the bifurcation $\tau>\tau_{0}$. Also $b_{2}<0$ and $T_{2}>0$, this implies that the bifurcating periodic solutions arising from $E_{3}\left(B^{*}, I^{*}, T^{*}, T_{c}^{*}\right)$ at $\tau_{0}$ are stable and the periods of limit cycle increases. 


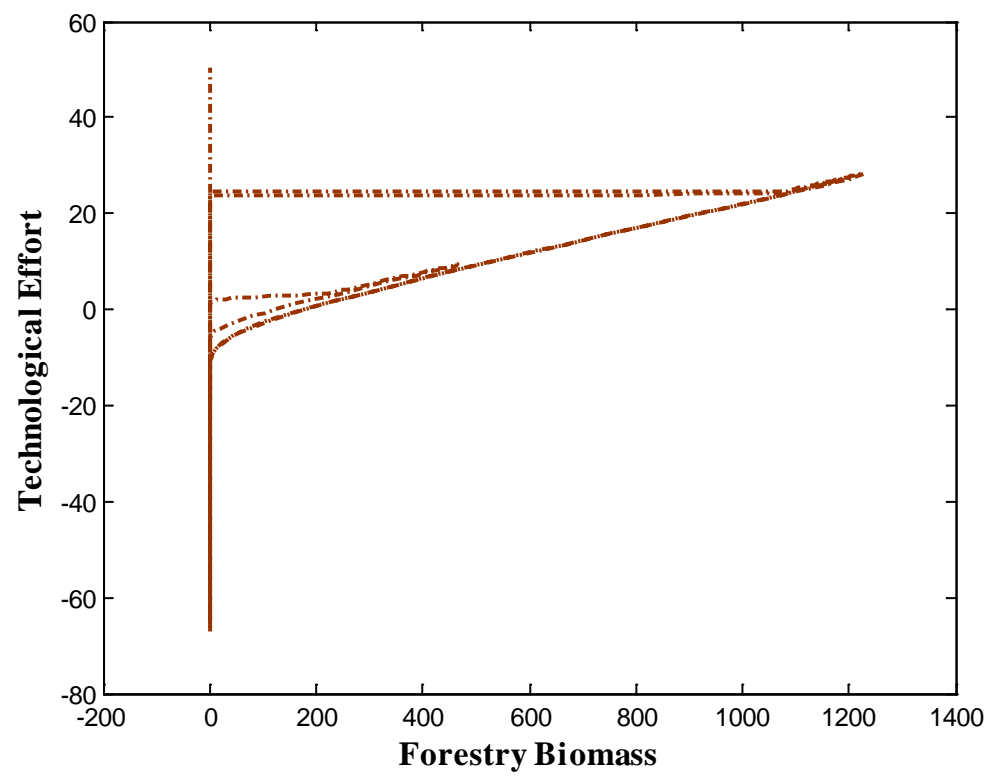

(a)

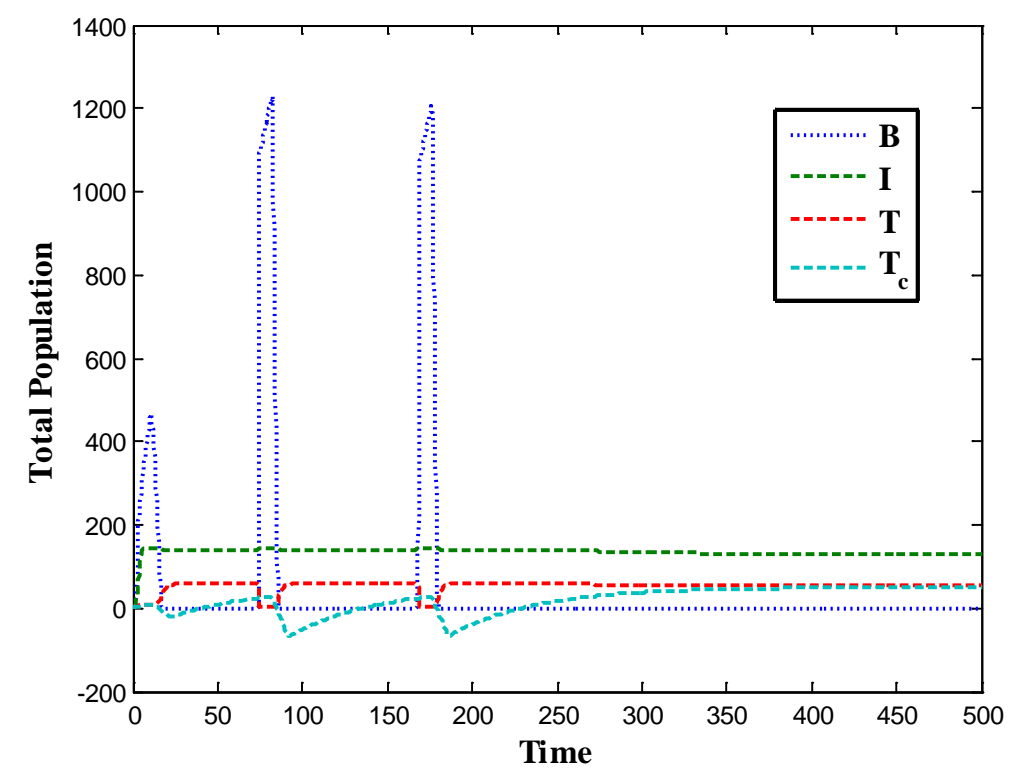

(b)

Figure 3. Trajectory portrait and phase portrait of system (4) for $\tau=8>\tau_{0}=5.82023$ and other parameters are same as (a).

\section{Conclusion}

In this paper, a nonlinear mathematical model is proposed and analyzed to see the effect of alternative resource and time delay on conservation of forestry biomass. We have obtained the explicit formulae that determine the stability and direction of the bifurcating periodic solutions by using the normal form theory and the center manifold theorem. For the given set of parameter values in (a), we found that, the Hopf bifurcation was supercritical with stable periodic solutions and the direction of bifurcation was $\tau>\tau_{0}$. Forests serve as a source of life for the forest based small and large scale industries. However, due to shrinking forests area, the industries are facing wood crisis. To overcome wood crisis, alternative resources like synthetic, liquid wood, plastic, wood composite lumber etc are good alternative for wood based products. For preserving our forestry biomass we can control the 


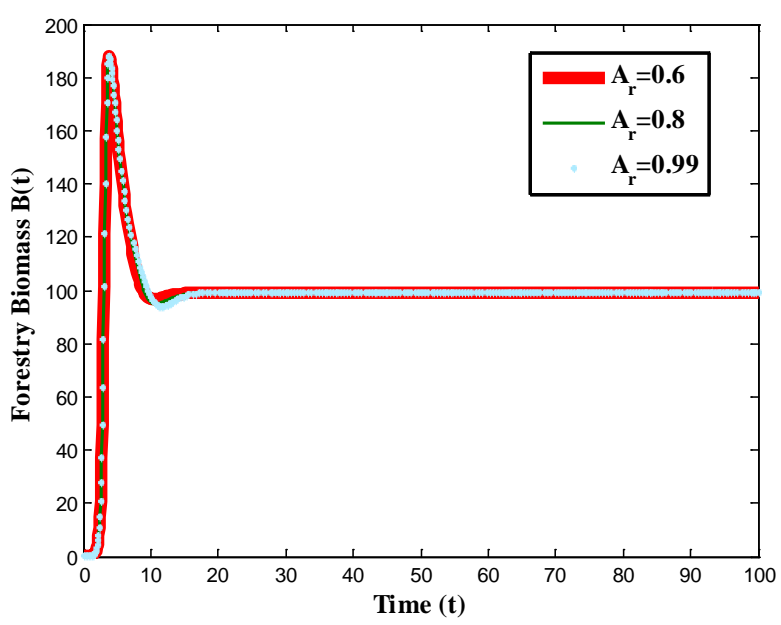

(a)

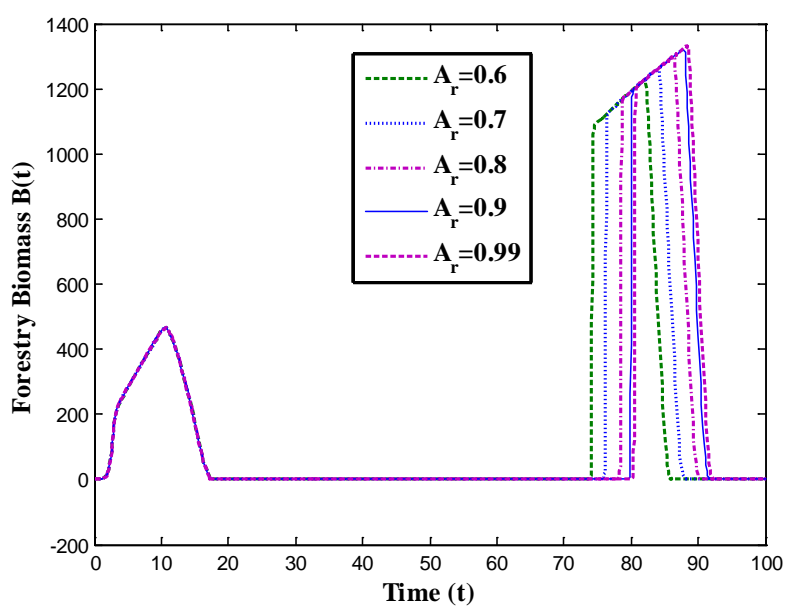

(b)

Figure 4. (a) and (b) show variation of the forestry biomass with time for different values of $A_{r}$ when $\tau=1<\tau_{0}=5.82023$ and $\tau=8>\tau_{0}=5.82023$ respectively and other parameter values are same as (a).

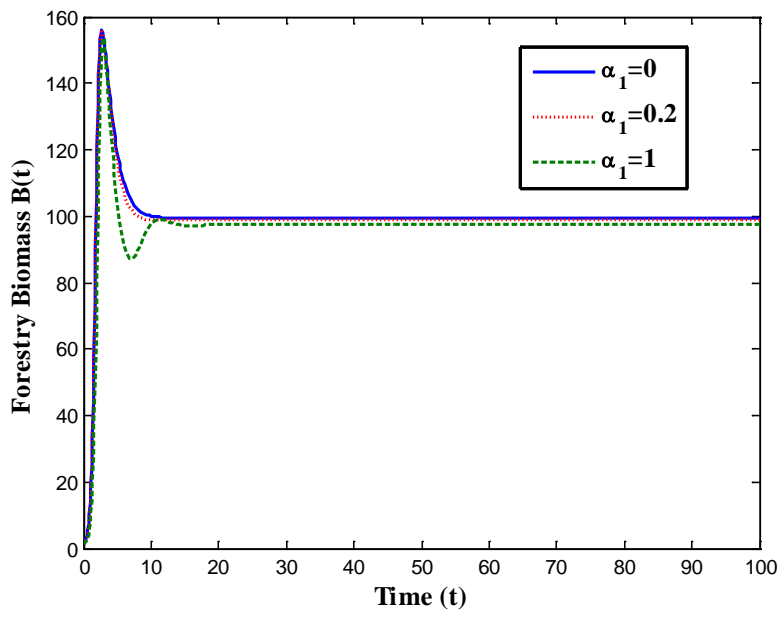

Figure 5. Variation of the forestry biomass with time for different values of $\alpha_{1}$ and other parameter values are same as (a). 


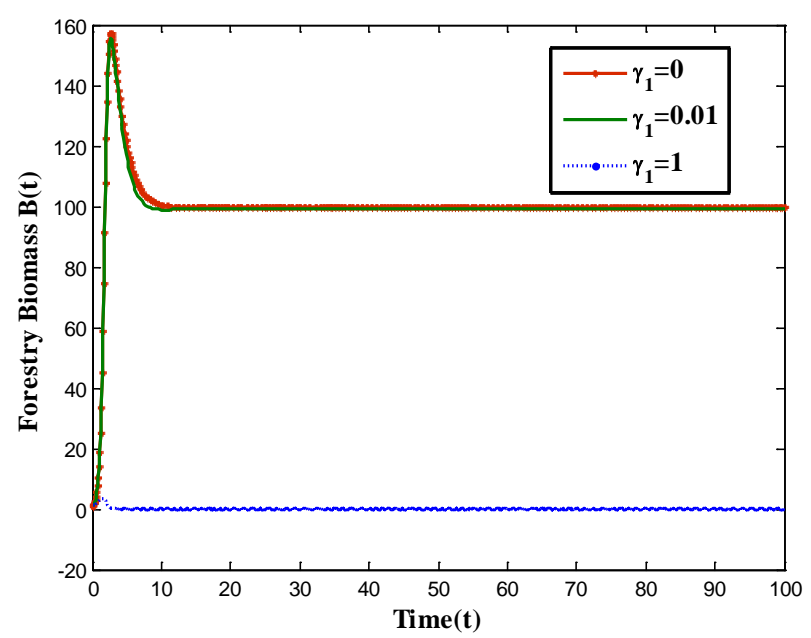

Figure 6. Variation of the forestry biomass with time for different values of $\gamma_{1}$ and other parameter values are same as (a).

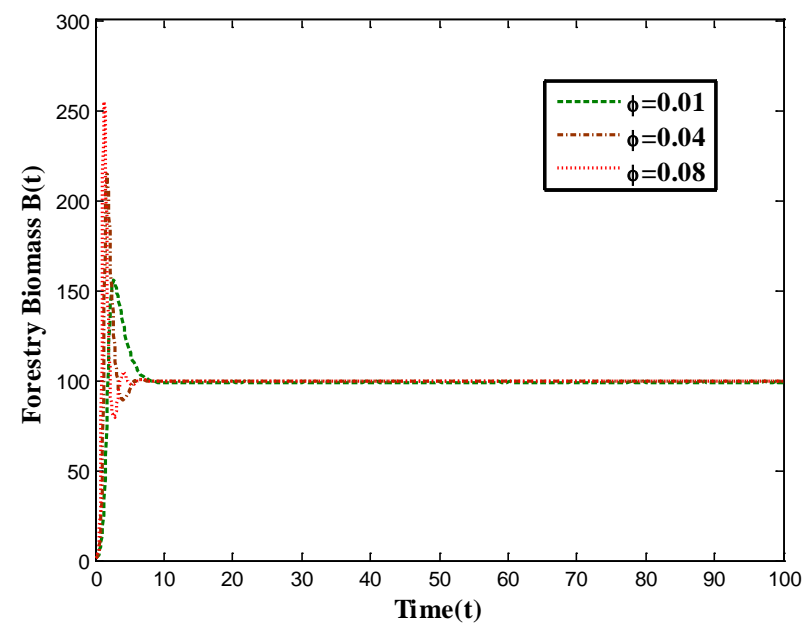

Figure 7. Variation of the forestry biomass with time for different values of $\phi$ and other parameter values are same as (a).

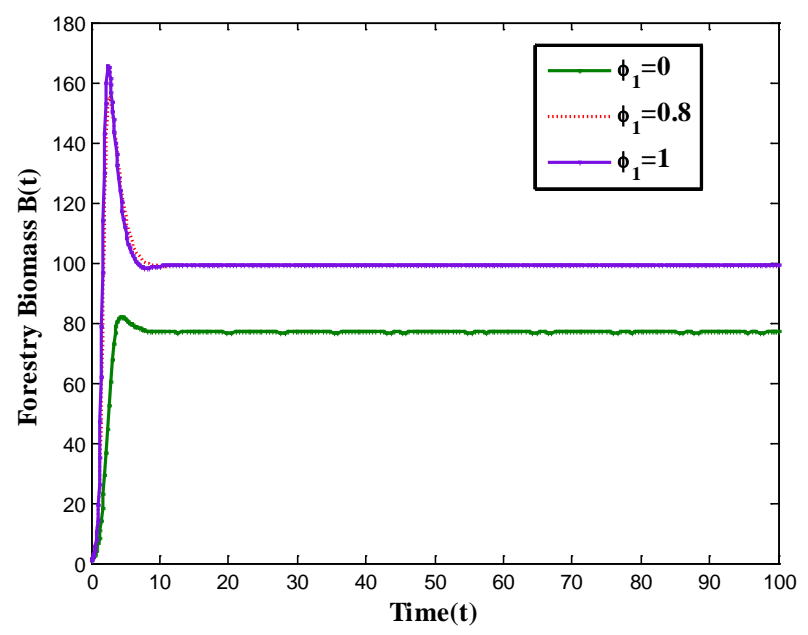

Figure 8. Variation of the forestry biomass with time for different values of $\phi_{1}$ and other parameter values are same as (a). 
wood based industries by human awareness or some government action. Hence, we conclude from our analysis that the forestry biomass may be conserved by applying technological effort and alternative resources.

\section{Acknowledgements}

Second author thankfully acknowledges the NBHM (2/40(29)/2014/R\&D-11/14138) for the financial assistance in the form of PDF.

\section{References}

[1] Shukla, J.B., Freedman, H.I., Pal, V.N., Misra, O.P., Agarwal, M. and Shukla, A. (1989) Degradation and Subsequent Regeneration of a Forestry Resource: A Mathematical Model. Ecological Modelling, 44, 219-229. http://dx.doi.org/10.1016/0304-3800(89)90031-8

[2] Li, W.Z., Ju, M.T., Liu, L., Wang, Y.N. and Li, T.L. (2011) The Effects of Biomass Solid Waste Resources Technology in Economic Development. Energy Procedia, 5, 2455-2460. http://dx.doi.org/10.1016/j.egypro.2011.03.422

[3] Shukla, J.B., Sharma, S., Dubey, B. and Sinha, P. (2009) Modelling the Survival of a Resource Dependent Population: Effect of Toxicants (Pollutants) Emitted from the External Sources as Well as Formed by Its Precursors. Nonlinear Analysis: Real World Applications, 10, 54-70. http://dx.doi.org/10.1016/j.nonrwa.2007.08.014

[4] Dubey, B. and Narayanan, A.S. (2010) Modelling Effects of Industrialization, Population and Pollution on a Renewable Resource. Nonlinear Analysis: Real World Applications, 11, 2833-2848. http://dx.doi.org/10.1016/j.nonrwa.2009.10.007

[5] Dhar, J., Chaudhary, M. and Sahu, G.P. (2013) Mathematical Model of Depletion of Forestry Resource, Effect of Synthetic Based Industries. International Journal of Biological, Life Science and Engineering, 7, 1-5.

[6] Misra, A.K. and Lata, K. (2013) Modeling the Effect of Time Delay on the Conservation of Forestry Biomass. Chaos, Solitons \& Fractals, 47, 1-11. http://dx.doi.org/10.1016/j.chaos.2012.10.002

[7] Misra, A.K., Lata, K. and Shukla, J.B. (2014) A Mathematical Model for Depletion of Forestry Resources Due to Population and Population Pressure Augmented Industrialization. International Journal of Modeling, Simulation and Scientific Computing, 5, 1-16.

[8] Misra, A.K., Lata, K. and Shukla, J.B. (2014) Effects of Population and Population Pressure on Forest Resources and Their Conservation: A Modeling Study. Environment, Development and Sustainability, 16, 361-374. http://dx.doi.org/10.1007/s10668-013-9481-x

[9] Agarwal, M. and Devi, S. (2011) A Resource-Dependent Competition Model: Effects of Toxicants Emitted from External Sources as Well as Formed by Precursors of Competing Species. Nonlinear Analysis: Real World Applications, 12, 751-766. http://dx.doi.org/10.1016/j.nonrwa.2010.08.003

[10] Hassard, B.D., Kzrinoff, N.D. and Wan, W.H. (1981) Theory and Application of Hopf Bifurcation: London Mathematics Society Lecture Note Series. Vol. 41, Cambridge University Press, Cambridge. 